Nonlinear Processes in Geophysics, 12, 671-689, 2005

SRef-ID: $1607-7946 / \mathrm{npg} / 2005-12-671$

European Geosciences Union

(c) 2005 Author(s). This work is licensed

under a Creative Commons License.

\title{
Statistical properties of nonlinear one-dimensional wave fields
}

\section{Chalikov}

Earth System Science Interdisciplinary Center (ESSIC), University of Maryland, College Park, MD 20 742-2465, USA

Received: 30 September 2004 - Revised: 31 January 2005 - Accepted: 21 March 2005 - Published: 30 June 2005

\begin{abstract}
A numerical model for long-term simulation of gravity surface waves is described. The model is designed as a component of a coupled Wave Boundary Layer/Sea Waves model, for investigation of small-scale dynamic and thermodynamic interactions between the ocean and atmosphere. Statistical properties of nonlinear wave fields are investigated on a basis of direct hydrodynamical modeling of 1-D potential periodic surface waves. The method is based on a nonstationary conformal surface-following coordinate transformation; this approach reduces the principal equations of potential waves to two simple evolutionary equations for the elevation and the velocity potential on the surface. The numerical scheme is based on a Fourier transform method. High accuracy was confirmed by validation of the nonstationary model against known solutions, and by comparison between the results obtained with different resolutions in the horizontal. The scheme allows reproduction of the propagation of steep Stokes waves for thousands of periods with very high accuracy. The method here developed is applied to simulation of the evolution of wave fields with large number of modes for many periods of dominant waves. The statistical characteristics of nonlinear wave fields for waves of different steepness were investigated: spectra, curtosis and skewness, dispersion relation, life time. The prime result is that wave field may be presented as a superposition of linear waves is valid only for small amplitudes. It is shown as well, that nonlinear wave fields are rather a superposition of Stokes waves not linear waves.

Potential flow, free surface, conformal mapping, numerical modeling of waves, gravity waves, Stokes waves, breaking waves, freak waves, wind-wave interaction.
\end{abstract}

\section{Introduction}

One of the most important problems of geophysical fluid dynamics is the interaction of wind waves and the atmospheric boundary layer. Until recently, the investigations of the marine boundary layer were based on the standard theory of

Correspondence to: D. Chalikov

(dchalikov@essic.umd.edu) a boundary layer above an infinite, flat, rigid surface (see review by Geernart, 1990). In fact, the presence of waves was considered a minor inconvenience forcing one to modify the roughness parameter affecting the wind profile. However, Wave Boundary Layer (WBL) has very specific properties, created by the presence of multi-mode moving surface. Waves modify the basic dynamic and thermodynamic interactions of air and water and, as well, the exchange by gases. Investigation of the interaction of wind and waves is important for parameterizing of micro-scale ocean-atmosphere interaction for weather and wind waves forecasting, and for climate modeling. This problem has been intensively investigated experimentally, theoretically, and numerically (see Chalikov, 1986; Donelan, 1990; Belcher and Hunt, 1998). All numerical investigations, based on Reynolds equations (begun by Gent and Taylor, 1976; Chalikov, 1978) were performed for 2-D turbulent flow above monochromatic waves, so they refer to an essentially steady motion. Application of monochromatic experimental and theoretical results for real nonlinear and multimode wave fields was based on a linear assumption. That is, all variables may be obtained by simple superposition of linear modes with different amplitudes. An evident extension of this monochromatic approach might be based on a multi-mode presentation of dispersive wave fields with preassigned spectrum $S$ (see review Chalikov, 1986). Generally, however, this presentation is also incorrect: with increasing the resolution of spectrum $\Delta k$, the geometrical properties of surface $\eta$ approximated by relation

$\eta=\sum\left(2 S_{k} \Delta k\right)^{1 / 2} \sin (k x-\omega t)$

depend essentially on $\Delta k$, and do not converge with $\Delta k \rightarrow 0$. The waves, assigned by (i) do not describe the typical shape of dominant waves, which usually have sharp crests and gentle troughs. It was establish long time ago in technical fluid mechanics that even small modifications of obstacles change the form drag significantly. Evidently, sharp-crested waves create form drag much larger that do smooth waves. Additionally, surface unsteadiness supports the strong nonsteadiness of both flow and pressure fields. This is presented correctly in perturbation theory only for very small amplitudes. A new approach was needed for generation of wave 
surfaces based on the principal equations for potential motion with an interface. Surely, actual sea waves are rotational. And they are affected by turbulence, because they are generated, migrate, and break in a shifted drift flow. However, potential approximation describes the wave dynamics better than (i), because it is not sensitive to resolution in Fourier space. Even in potential approximation, the wave surface appears as a real physical object, whereas spectral presentation obscures its nonlinear nature. Actually, as resolution $\Delta k$ increases, the different $k$-th modes assigned in the initial conditions do not move as separate wave modes with specific dispersion relation: waves separated in a Fourier space join together, forming a superposition of nonlinear waves. The spectrum therefore describes a finite number of nonlinear waves rather than a continuous spectrum of linear waves. The experimental and simulated spectra appear continuous, because of the essential linearity of Fourier analysis and because of the amplitudes of these discrete nonlinear waves change in time. Contrary to (i), the solution of the wave equations converges with increasing spectral resolution (Chalikov and Sheinin, 1995), what proves the applicability of a such approach to the simulations of wind-wave interaction.

This study describes the results of numerical simulations of multi-mode wave fields based on a scheme developed by Chalikov and Sheinin (1996, 1998, 2005). The air/water model has been also completed (Chalikov, 1998); and numerical investigation of wind-wave interactive dynamics is underway. Because wave development of waves occurs at distances which are much larger than the length of the dominant wave, the periodic boundary conditions have been used. This assumption simplifies the construction of a numerical scheme, because it makes possible the application of the Fourier transform method. In this paper, we consider 1-D nonlinear waves only. The scheme here developed is exact, allowing simulating of waves for periods much longer than the period of the dominant wave.

The primary advantage of the potential approximation is that the system of Euler equations is reduced to the Laplace equation. However, the solution to the flow problem of surface wave motion is complicated by the required application of kinematics and dynamic boundary conditions (both nonlinear) onto the free surface, the location of which is unknown at any given time, $t$.

Numerical simulation of surface waves has a long history. The most general approach to simulate a motion with a free surface is based on a marker and cell (MAC) method (Harlow and Welch, 1965). This approach assumes the tracing of a variable surface within a fixed grid with different order accuracy (for example, Noh and Woodward, 1976; Hirt and Nichols, 1981; Prosperetti and Jacobs, 1983; Miyata, 1986). Currently, the applicability of this method is restricted to simulations over relatively short-term periods. Accuracy of this method will increase significantly, however, when very high resolution becomes possible. An advantage of this method is that it can be used for simulation of 3-D rotational motion of viscous fluids even for non-single value interface. A motion with a single-value 1-D and 2-D interface is readily simulated using the simplest surface-following coordinates $(x, y, z-\eta(x, y))$, where $(x, y, z)$ are Cartesian coordinates and $\eta$ is surface elevation (Chalikov, 1978). This system of coordinates is unsteady and non-orthogonal, so equations of motion become complicated. Still, this method has been applied for the simulation of wave interaction with a shear flow (Dimas and Triantafyllou, 1994). Evidently, this approach may be combined with the MAC method, applied locally in the intervals with large steepness. Waves on finite depth have been investigated by transforming the volume occupied by fluid into a rectangular domain (Dommermuth et al., 1993). Much more complicated surface-following transformations have been constructed, including a case of a multiple-valued surface (Thompson et al., 1982). The grid method was generalized with adaptive grids (e.g. Fritts et al., 1988), and using a finite-volume approach (Farmer et al. (1993).

Fortunately, many observed properties of surface waves are reproduced well based on a potential approach, which makes possible a reduction in dimensionality by one. The numerical methods for inviscid free-surface flow have been reviewed by Mei (1978), Yeung (1982), Hyman (1984), and for viscous flows by Floryan and Rassmussen (1989). The most recent review of numerical methods for incompressible nonlinear free-surface flow was presented by Tsai and Yue (1996). The scope of this review is limited to those works published after the last-mentioned review, which is devoted to free periodic waves and is based on the principal equation for potential waves.

The simulation of nonlinear unsteady potential flow with a free surface began with the development of the EulerianLagrangian boundary integral equations by Longuet-Higgins and Cokelet (1976) for steep over-turning waves. Wave instability was generated by asymmetric pressure applied on a surface. This method, in principle, may be generalized for 3-D motion, but it demands considerable computational resources. A boundary method based on the Cauchy integral formulation for 2-D problem was developed by Vinje and Brevig (1981), Baker et al. (1982) and by Roberts (1983). This method was used by Tanaka et al. (1987), to study the instability and breaking of a solitary wave. Dommermuth et al. (1998) compared the solution based on the Cauchy integral method with precise measurements in an experimental wave tank. Good agreement was obtained. The boundary integral method was extended by Dold and Peregrine (1986, hereafter DP); they constructed a precise scheme for performing simulations of wave evolution with good conservation of invariants. A detailed description of this method was given by Dold (1992). Stability was confirmed by simulations of steep Stokes wave for several wave periods. This scheme was used successfully for simulation of nonlinear group effects (Henderson, et al., 1999); of dynamics of steep forced waves (Bredmose et al., 2003), and for investigating the onset of breaking (Banner and Tian, 1996, 1998; Song and Banner, 2002). An advantage of this model is extreme efficiency: reliable results can be obtained with low resolution using modest computer resources. For short-term periods, is possible to distribute the points non-uniformly; thus 
allowing the model to reproduce sharpening and overturning waves. To increase stability, the authors applied very selective smoothing. Dold (1991) noted, however, that the advantage conferred by non-uniformity of the grid is absent for long-term integration. Wave sharpening results from nonlinear group effects, which generate a convergence of energy in physical space (Song and Banner, 2002), though is impossible to predict the location of these events. Nonetheless, Henderson et al. (1999) demonstrated good results from longterm simulations of nonlinear evolution of wave field with an initially uniform grid.

Another group of numerical methods is based on traditional perturbation expansions (often combined with Fourier transform method); in principle, these include arbitrary high orders of interactions (Watson and West, 1975; Dommermuth and Yue, 1987; West et al., 1987). However, the number of needed Fourier modes in this scheme multiplies with increasing steepness. Indeed, this method becomes inapplicable when waves approach overturning. Modification of the high-order spectral method was suggested by Fenton and Rienecker (1982).

Craig and Sulem (1993) improved stability by using the expansion for vertical velocity instead of potential. This method was later generalized for 2-D potential waves (Bateman et al., 2001). A numerical scheme for 1-D potential waves, based on non-orthogonal surface-following coordinate system and Fourier transform, was developed by Chalikov and Liberman (1991). The method is based on iterative transfer of the potential from a fixed coordinate system onto free surface; it was used to simulate the bound waves dynamics observed by Yuen and Lake (1982). This method is also applicable to 2-D potential waves; however, it becomes ineffective for large numbers of modes with highly variant amplitudes (also true for many methods based on expansions). The reason for this limitation restriction: the small waves overrun the surfaces of large waves. The amplitudes of wave disturbances with large wave numbers attenuate with depth quickly, becoming insignificant for the depth of dominant wave height (Zhang et al., 1993). In this case, restoring the high-order modes on a free surface becomes inaccurate. Still, the high- order perturbation methods (and all methods based on the surface-following coordinates) represent a huge advance over quasi-linear theories based on small-amplitude assumption.

A numerical scheme for direct hydrodynamical modeling of 1-D nonlinear gravity and gravity-capillary periodic waves was developed by Chalikov and Sheinin $(1996,1998)$. This scheme is based on conformal mapping of a finitedepth water domain. For the stationary problem, this mapping represents the classical complex variable method (e.g. Crapper, 1957, 1984) originally developed by Stokes (1847). In a stationary problem, this method employs the velocity potential $\Phi$ and the stream function $\Psi$ as the independent variables. A nonstationary conformal mapping was introduced also by Whitney (1971), then considered by Kano and Nishida (1979) and by Fornberg (1980). Tanveer (1991, 1993) used this approach for investigating Rayleigh-Taylor instability and the generation of surface singularities. A new way of deriving equations, a description of a numerical scheme (and its validation) as well as the results of long-term simulations were presented at ONR meeting held in Arizona in 1994 (Sheinin, Chalikov, 1994). Later, this scheme was described in detail by Chalikov and Sheinin $(1996 ; 1998)$ and in Sheinin and Chalikov (2000). The ChSh numerical approach is based on nonstationary conformal mapping for finite depth. This allows rewriting of the principal equations of potential flow with a free surface in a surface-following coordinate system. The Laplace equation retains its form, and the boundaries of the flow domain (i.e. the free surface and, in the case of finite depth, the bottom) are coordinate surfaces in the new coordinate system. Accordingly, the velocity potential in the entire domain receives a standard representation based on its Fourier expansion on the free surface. As a result, the hydrodynamical system (without any simplifications) is represented by two relatively simple evolutionary equations that can be solved numerically in a straightforward way. The advantages of this approach were briefly discussed by Dyachenko et al. (1996); later, the method was used by Zakharov et al. (2002) to demonstrate the nonlinear properties of steep waves. In principle, the ChSh method is similar to method developed by Meiron et al. (1981). They concluded that this method is applicable only to "moderately distorted geometry". For simulations of the Stokes wave with peak-to-trough amplitude at $80 \%$ of the theoretical maximum, they found that "time stepping errors can cause modulation of the steady waves for times longer than $t=4 \pi$ " (time is normalized with the length scale and gravity acceleration). Our scheme, in contrast, allows simulation of the propagation of Stokes wave with amplitude at $98 \%$ of the maximum for hundreds of periods (and much longer) without noticeable distortions (see Fig. 1, below). Because Meiron et al. (1981) used the same accuracy time-stepping scheme as the 4th order Runge-Kutta scheme applied here, we conclude that their errors were generated simply by low resolution: for an approximation of Stokes wave profile only $N=64$ points were used. We exploited thousands of points. (Zakharov et al. (2002) used up to one million Fourier modes). Imprecise approximation of the initial shape of a Stokes wave also might result in instability. The stability of Stokes wave depends largely on the degree of truncation of the Fourier series used to describe the wave. This effect is better pronounced for large steepness. For example, for the case $a=0.42$ (half of crest-to-trough height), the Stokes wave initially assigned by five modes (with resolution 2000 modes, 8000 grid points) collapses to time $\mathrm{t}=1$. Exact Stokes wave (assigned by the hundreds of modes) runs stably for the same resolution the thousands of periods.

Note that a model based on high resolution is very fast, so most problems can be investigated with a personal computer.

This model is a rarity in geophysical fluid dynamics when the equations describing the real process can be solved with unusual accuracy (see Fig. 1). This statement is fully correct if steepness is not high. Increasing local steepness often results in a developing instability thence overturning 


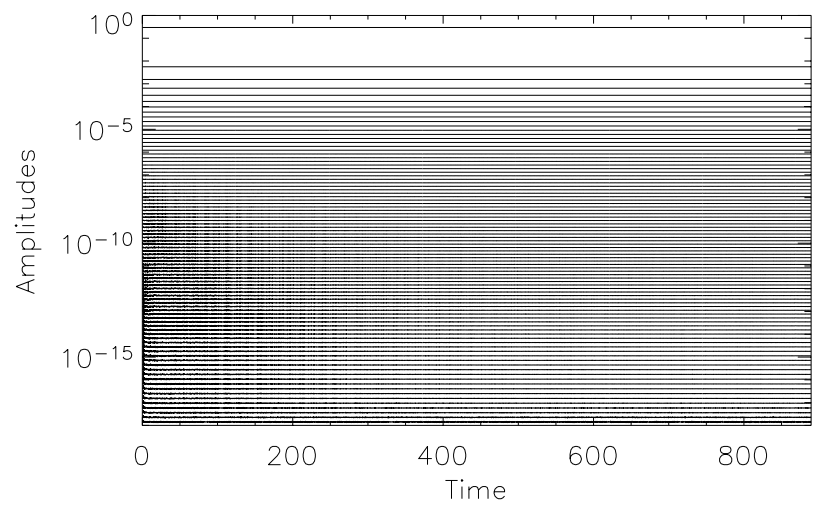

Fig. 1. Long-term evolution of amplitudes of the first 880 constituents of the Stokes waves: ( $\mathrm{ak}=0.42$; each 10 th constituents is shown) during 2686500 time steps (932 periods).

of sharp crests. Formally, conformal mapping exists up to the moment when an overturning volume of water touches the surface (Dyachenko, 2004, personal communication). In such an evolution, the number of Fourier modes needed increases very quickly. If the special measures (smoothing) are not taken, the calculations typically terminate much earlier, due to strong crest instability (Longuet-Higgins, 1996) manifested by splitting of the falling volume into two phases. This phenomenon is obviously non-potential. Hence, as in many branches of geophysical fluid dynamics, special measures must be taken to prevent numerical instabilities while concurrently accounting on the physical consequences of these events.

Our method is applied to the simulation of wave fields represented in initial conditions by superposition of running waves with random phases assigned by linear theory. We show that wave fields lose their initial structure and soon develop new, specific features. These become pronounced the larger the nonlinearity of the initial state. Of course, this developing of waves influences significantly the air flow above wave surface.

\section{Equations}

Consider the principal 2-D equations for potential waves written in Cartesian coordinates, i.e. the Laplace equation for the velocity potential $\Phi$

$\Phi_{x x}+\Phi_{z z}=0$,

and the two boundary conditions at the free surface $h=h(x, t)$ : the kinematic condition

$h_{t}+h_{x} \Phi_{x}-\Phi_{z}=0$,

and the Lagrange integral

$\Phi_{t}+\frac{1}{2}\left(\Phi_{x}^{2}+\Phi_{z}^{2}\right)+h+p_{e}-\sigma h_{x x}\left(1+h_{x}^{2}\right)^{-\frac{3}{2}}=0$,

where $p_{e}$ is the external surface pressure. (Independent variables in subscripts denote partial differentiation with respect to these variables.)
The equations are to be solved in the domain

$-\infty<x<\infty-H \leq z \leq h(x, t)$,

where $H$ is either a finite depth or infinity. The variables $\Phi$ and $h$ are considered to be periodic with respect to $x$ :

$\Phi(x+2 \pi, z, t)=\Phi(x, z, t) h(x+2 \pi, t)=h(x, t)$,

and a normal velocity condition at the bottom is assumed to be zero:

$\Phi_{z}(x, z=-H, t)=0$

Equations (1)-(3) are written in the nondimensional form with the following scales: length $L$, where $2 \pi L$ is the (dimensional) period in the horizontal, time $L^{1 / 2} g^{-1 / 2}$ and the velocity potential $L^{3 / 2} g^{-1 / 2}$ ( $g$ is the acceleration of gravity). Pressure is taken to be normalized by water density (so its scale is Lg). The last term in Eq. (3) describes the effect of surface tension, and

$\sigma=\frac{\Gamma}{g L^{2}}$

is a nondimensional parameter $\left(\Gamma \approx 8 \cdot 10^{-5} \mathrm{~m}^{3} \mathrm{~s}^{-2}\right.$ is the kinematic coefficient of surface tension for water).

System in Eqs. (1)-(6) is solved as an initial-value problem for the unknown functions $\Phi$ and $h$, with initial conditions $\Phi(x, z=h(x, t=0), t=0)$ and $h(x, t=0)$. Note that although Eqs. (2) and (3) are for the free surface, there are no straightforward ways to reduce the problem to a 1-D because, to evaluate $\Phi_{z}$, one has to solve the Laplace equation (1) in the domain (4) with a curvilinear upper boundary that may be any function of $x$. This difficulty is known to render integration of the system in Cartesian coordinates either insufficiently accurate or too expensive computationally (Chalikov and Liberman, 1991). So, for time periods much greater than the time scale, it is quite impractical.

To realize a feasible numerical solution, we introduce a time-dependent surface-following coordinate system that conformally maps the original domain (4) onto the strip

$-\infty<\xi<\infty-\tilde{H} \leq \zeta<0$

with a periodicity condition given as

$x(\xi, \zeta, \tau)=x(\xi+2 \pi, \zeta, \tau)+2 \pi$,

$z(\xi, \zeta, \tau)=z(\xi+2 \pi, \zeta, \tau)$

where $\tau$ is the new time coordinate $\tau=t$.

According to complex variable calculus, conformal mapping $(7) \rightarrow(4)$ exists and is unique up to an additive constant for $x$. Note that for the stationary problem, this mapping represents the classic complex variable method (e.g. Crapper, 1984) originally developed by Stokes (1847)

Due to periodicity condition (8), the required conformal mapping can be represented by the Fourier series:

$x=\xi+x_{0}(\tau)+\sum_{-M \leq k<M, k \neq 0} \eta_{-k}(\tau) \frac{\cosh k(\varsigma+\tilde{H})}{\sinh k \tilde{H}} \vartheta_{k}(\xi)$, 


$$
z=\zeta+\eta_{0}(\tau)+\sum_{-M \leq k<M, k \neq 0} \eta_{k}(\tau) \frac{\sinh k(\varsigma+\tilde{H})}{\sinh k \tilde{H}} \vartheta_{k}(\xi),
$$

where $\eta_{k}$ are the coefficients of Fourier expansion of the free surface $\eta(\xi, \tau)$ with respect to the new horizontal coordinate $\xi$ :

$\eta(\xi, \tau)=h(x(\xi, \zeta=0, \tau), t=\tau)=\sum_{-M \leq k \leq M} \eta_{k}(\tau) \vartheta_{k}(\xi)$,

$\vartheta_{k}$ denotes the functions

$\vartheta_{k}(\xi)=\left\{\begin{array}{l}\cos k \xi, k \geq 0 \\ \sin k \xi, k<0\end{array} ;\right.$

$M$ is the truncation number (so far $M=\infty$ is assumed); $x_{0}(\tau)$ can be chosen arbitrarily, though it is convenient to assume that $x_{0}(\tau)=0$.

Nontraditional presentation of the Fourier transform with definition (12) is, in fact, more convenient for calculations because $\left(\vartheta_{k}\right)_{\xi}=k \vartheta_{-k}$ and $\sum\left(A_{k} \vartheta_{k}\right)_{\xi}=-\sum k A_{-k} \vartheta_{k}$. So the Fourier coefficients $A_{k}$ form the array $A(-M: M)$, thus making possible a compact programming in Fortran90.

The lower boundary $\zeta=-\sim H$ can not be chosen arbitrarily, since the relation

$z(\xi, \zeta=-\tilde{H}, \tau)=-H$

must hold; after substituting expansion (10), (13) yields:

$\tilde{H}=H+\eta_{0}(\tau)$.

Since $\eta_{0}$ is determined by the Fourier expansion (11), and, generally, is an unknown function of time, $\tilde{H}$ also is timedependent. Note that the definition of both coordinates $\xi$ and $\zeta$ is based on Fourier coefficients for surface elevation. It follows then from (9) and (10), that the time derivatives $z_{\tau}$ and $x_{\tau}$ for Fourier components are connected by the simple relation

$$
\left(x_{\tau}\right)_{k}=\left(z_{\tau}\right)_{-k} \operatorname{coth}(k \tilde{H}),
$$

Due to conformity of the mapping, Laplace equation (1) retains its form in $(\xi, \zeta)$ coordinates. It is shown in ChSh that the system (1) - (3) can be written in the new coordinates as follows:

$$
\begin{aligned}
& \Phi_{\xi \xi}+\Phi_{\zeta \zeta}=0 \\
& z_{\tau}=x_{\xi} g+z_{\xi} f \\
& \Phi_{\tau}=f \Phi_{\xi}-\frac{1}{2} J^{-1}\left(\Phi_{\xi}^{2}-\Phi_{\zeta}^{2}\right)-z-p_{e}+ \\
& \quad \sigma J^{-3 / 2}\left(-x_{\xi \xi} z_{\xi}+z_{\xi \xi} x_{\xi}\right),
\end{aligned}
$$

where (17) and (18) are written for the surface $\zeta=0$ (so that $z=\eta$, as represented by expansion (11)), $J$ is the Jacobian of the transformation

$J=x_{\xi}^{2}+z_{\xi}^{2}=x_{\zeta}^{2}+z_{\zeta}^{2}$,

$g=\left(J^{-1} \Phi_{\zeta}\right)_{\zeta=0}$; and $f$ is a generalization of the Hilbert transform of $g$, which for $k \neq 0$ may be defined in Fourier space as

$f_{k}=g_{-k} \operatorname{coth} k \tilde{H}$,

following in fact from Eq. (15).

Boundary condition (6) is rewritten as

$\Phi_{\zeta}(\xi, \zeta=-\tilde{\mathrm{H}}, \tau)=0$.

The solution to the Laplace equation (16) with boundary condition (22) is readily yields to Fourier expansion, which reduces the system (16)-(18) to a 1-D problem:

$\Phi=\sum_{-M \leq k \leq M} \phi_{k}(\tau) \frac{\cosh k(\zeta+\sim H)}{\cosh k \sim H} \vartheta_{k}(\xi)$,

where $\phi_{k}$ are Fourier coefficients of the surface potential $\Phi(\xi, \zeta=0, \tau)$. Thus, Eqs. (17), (18), and (20) constitute a closed system of prognostic equations for the surface functions $z(\xi, \zeta=0, \tau)=\eta(\xi, \tau)$ and $\Phi(\xi, \zeta=0, \tau)$. It may also be regarded as a system of ordinary differential equations for the Fourier coefficients $\eta_{k}, \phi_{k}$. Its explicit form would be somewhat complicated; however, it is not needed. As described in the next section, we use the Fourier transform method to calculate the nonlinearities. Indeed, the transform method may be called an exact method if the order of nonlinearity is finite. Otherwise, the accuracy of the method should be established empirically.

Thus, the original system of Eqs. (1)-(3) is transformed into two evolutionary Eqs. (17) and (18) which are written for the free surface and, thus, are essentially 1-D (both spatial derivatives of $\Phi$ are obtained by differentiating the series (23)) and can be solved using the Fourier transform method (see next Sect. 3 and also ChSh).

For deep water $(H=\infty)$, the coefficients in expansions (9), (10) and (23) become simpler. The domain (7) turns into the semi-plane $\zeta<0(z \rightarrow-\infty$ when $\varsigma \rightarrow-\infty$, a condition which replaces (14)); and the conformal mapping (9) and (10) acquires the form

$$
\begin{gathered}
x=\xi+x_{0}(\tau)+\sum_{-M \leq k<M, k \neq 0} \eta_{-k}(\tau) \exp (k \zeta) \vartheta_{k}(\xi), \\
z=\zeta+\eta_{0}(\tau)+\sum_{-M \leq k<M, k \neq 0} \eta_{k}(\tau) \exp (k \zeta) \vartheta_{k}(\xi) ;
\end{gathered}
$$

and the solution (23) of the Laplace equation (16) reduces to

$\Phi=\sum_{-M \leq k \leq M} \phi_{k}(\tau) \exp (k \zeta) \vartheta_{k}(\xi) ;$

In the absence of capillarity, the upper boundary condition is $p=p_{e}$. All model simulations presented herein hold the prescribed external surface pressure constant. One may therefore assume

$p(\xi, \zeta=0, \tau)=p_{e}=0$. 
The lower boundary condition readily follows from the Lagrange integral, written for the bottom in the (nondimensional) Cartesian coordinates: $p_{z}=-1$. So, in the new coordinates we have

$z_{\zeta} p_{\zeta}=-J$ at $\zeta=-\tilde{H}$.

For deep water, this condition becomes

$p_{\zeta} \rightarrow-1$ at $\zeta \rightarrow-\infty$.

\section{Numerical scheme and its accuracy}

For spatial approximation of system (32) and (33) we use a Galerkin-type (or "spectral") method based on a Fourier expansion of the prognostic variables with a finite truncation number $M$. The problem is thus reduced to a system of ordinary differential equations for $4 M+2$ Fourier coefficients

$\eta_{k}(\tau), \phi_{k}(\tau),-M \leq k \leq M:$

$\dot{\eta}_{k}=E_{k}\left(\eta_{-M}, \eta_{-M+1}, \ldots, \eta_{M}, \phi_{-M}, \phi_{-M+1}, \ldots, \phi_{M}\right)$

$\dot{\phi}_{k}=F_{k}\left(\eta_{-M}, \eta_{-M+1}, \ldots, \eta_{M}, \phi_{-M}, \phi_{-M+1}, \ldots, \phi_{M}\right)$

where $E_{k}, F_{k}$ are, respectively, the Fourier expansion coefficients for the right-hand sides of Eqs. (17) and (18) as functions of $\xi$.

To calculate $E_{k}, F_{k}$ as functions of the prognostic variables $\eta_{k}, \phi_{k}$, differentiation of the Fourier series is used (the spatial derivatives are thus evaluated exactly) and the nonlinearities are calculated with the so-called transform method (Orszag, 1970; Eliassen et al.., 1970), by their evaluation on a spatial grid. If $Y(u(\xi), v(\xi), w(\xi), \ldots)$ is a nonlinear function of its arguments represented by their Fourier expansions, grid-point values $u\left(\xi_{j}\right), v\left(\xi_{j}\right), w\left(\xi_{j}\right), \ldots$ are first calculated (in other words, inverse Fourier transforms are performed).Then $Y^{(j)}=Y\left(u\left(\xi^{(j)}\right), v\left(\xi^{(j)}\right), w\left(\xi^{(j)}\right), \ldots\right)$ are evaluated at each grid point. Finally, the Fourier coefficients $Y_{k}$ of the function $Y$ are found by a direct Fourier transform. Here $\xi^{(j)}=2 \pi(j-1) / N-N$ is the number of grid-points. This approach is largely exploited extensively in geophysical hydrodynamics, in global atmospheric modeling particularly.

For the method to be a purely Galerkin one, that is, to ensure the minimum mean square approximation error, the Fourier coefficients $E_{k}, F_{k}$ must be evaluated exactly for $-M \leq k \leq M$. For this purpose, one must choose

$N>(v+1) \mathrm{M}$

where $v$ is the maximum order of nonlinearities. Since the right-hand sides of Eqs. (17) and (18) include division by the Jacobian, the nonlinearity is of infinite order such that the above condition on $N$ can not be met. However, numerical integrations show that if one chooses a value of $N$ ensuring exact evaluation of cubic nonlinearities ( $v=3$ in Eq. (32)), a further increase in $N$ (with a fixed $M$ ) does not affect the numerical solution. For the results presented in Section 5, $N=4 M$ was chosen.
However high the spectral resolution might be, long-term simulations of strongly nonlinear waves require that the energy flux be parameterized into the severed part of the spectrum $(|k|>M)$. If ignored, spurious energy accumulations at large wave numbers can corrupt the numerical solution. Simple dissipation terms were therefore added to the right-hand sides of Eqs. (43) and (44):

$\dot{\eta}_{k}=E_{k}-\mu_{k} \eta_{k}$

$\dot{\phi}_{k}=F_{k}-\mu_{k} \phi_{k}$

with

$\mu_{k}= \begin{cases}r M\left(\frac{|k|-k_{d}}{M-k_{d}}\right)^{2} & \text { if }|k|>k_{d} \\ 0 & \text { otherwise }\end{cases}$

where $k_{d}=M / 2$ and $r=0.25$ were chosen for all runs discussed below. We found the sensitivity of the results to reasonable variations of $k_{d}$ and $r$ to be low. The dissipation effectively absorbs energy at wave numbers close to the truncation number $M$ while leaving longer waves virtually intact and as well as modes with wave numbers $|k| \leq k_{d}$ unaffected. Note that an increase of the truncation number $M$ shifts the dissipation area to higher wave numbers (and, with $M \rightarrow \infty$, the energy sink due to dissipation tends to zero). Therefore, the scheme with the dissipation incorporated retains an approximation of the original (non-dissipative) system.

For time integration, the fourth-order Runge-Kutta scheme was used. The choice of time step was done empirically. For example, for $M=100$, a time step was equal to 0.01 . For $\mathrm{M}=1000$, it was 0.002 . Increasing local steepness and surface curvature often forces to apply smaller time steps. Capillarity effects were not included in this study. If these are taken into account, the restrictions on time step become more severe (ChSh).

A separate problem is initial data normally given in the Cartesian coordinates. These need to be converted to the $(\xi, \zeta)$ coordinates. For this purpose, and for processing of the results, an algorithm based on the periodic high-order spline functions has been developed. The algorithm carries out the transformation with computer accuracy.

The effective method of validating the numerical scheme is to compare the results of integration of the nonstationary Eqs. (16)-(20) (or, equivalently, Eqs. (1)-(3)) with the analytical solution obtained in a moving coordinate system. Only for Crapper's waves (Crapper, 1957) such solutions can be written analytically. Other stationary solutions, such as Stokes waves (progressive, pure gravity waves on deep water) have previously been obtained with various degrees of approximation. The most accurate calculations of Fourier coefficients for Stokes waves were performed by Drennan, et al. (1988). To obtain stationary solutions with computer accuracy (crucial for model validation), we developed an iterative algorithm that is based on operators of integration and generalized Hilbert transformation in Fourier space. It employs the Fourier transform method to calculate nonlinearities. This algorithm for calculation gravity and capillary 
gravity waves for deep water was described in detail in ChSh (Sect. 3). The results for the case of finite depth were obtained in Sheinin and Chalikov (2000). These solutions (as well as Crapper's waves) may be considered exact. They were used for validation of the nonstationary model (system Eqs. (16)-(18)).

Discussion of long-term validation runs is found in ChSh. The model was validated against the three types of waves: pure capillary deep-water (Crapper's) waves (analytical solutions), pure gravity, and also gravity-capillary waves, the latter obtained numerically with the algorithm referred to above. For all test cases, visual comparison of instantaneous wave profiles showed that the waves moved with no any spurious perturbations. To estimate "steadiness" of the numerical solution quantitatively, we calculated the phase velocities and amplitudes of the Fourier components for consecutive moments of time, and obtained their temporal means and standard deviations over the period of integration. Results in ChSh (Table 4) show that even for steep waves the calculated phase velocities were very close to their exact values (i.e. those obtained for the stationary solutions) for all three types of waves. Conservation of the amplitudes was also very accurate: deviations during the simulations from initial values were always less than $10^{-7}$ for the Stokes wave, and less than $10^{-11}$ for the capillary and gravity-capillary waves. The modes retained their initial energies and remained consistent in phase; consequently, the simulated waves did not change their shapes during the integration. This confirms that these waves are stable with respect to truncation errors, and also that the numerical solutions approximate the solutions of the original differential equations with high accuracy. For all model simulations described in (Chalikov and Sheinin, 2005), differences between the solutions presented and their versions obtained with $M$ twice as large were nearly absent, confirming a convergence of numerical scheme.

Additional validation was performed by simulating of very steep Stokes waves for $a k=0.42(M=1000, \Delta \tau=0.0025)$. The evolution of first 880 amplitudes is shown in Fig. 1. This boring picture shows that amplitudes, and, consequently, the shape of Stokes wave remained unchanged with very high accuracy. These integrations can be continued much longer without of noticeable changes in amplitudes. After 2686500 time steps (932 periods), the total energy for $a k=0.42$ and $M=1000$ decreased merely at $3 \cdot 10^{-8} \%$. The analogous calculations for $a k=0.42$ performed by Dold (1992) quickly collapsed due to numerical instability. Exact phase velocity of Stokes waves with ak $=0.42$, obtained for the stationary solution is 1.089578 . Direct calculation of phase velocity of simulated Stokes wave gave value $1.089579 \pm 10^{-6}$.

Note that validation based on simulation of running Stokes waves is complete and not trivial, because nonstationary equations "do not know" the stationary solution obtained in a moving coordinate system via a different method. However, the results shown in Fig. 1 were obtained for demonstration of accuracy of scheme only. High stability of steep Stokes wave was determined by its position in a Fourier space: the modes were connected with wave numbers $k=1,2,3 \ldots M$.
So, all Fourier modes were the modes of Stokes wave, and there was no room for developing of an instability. Another situation takes place when the modes of the Stokes wave are the Fourier modes with $k=n, 2 n, 3 n \ldots M$ ( $n$ is any integer number), and the intermediate modes in initial conditions equal to zero. In this case, the errors of approximation can initiate the Benjamin-Feir instability (Benjamin, Feir, 1967), and a train of the Stokes waves can loose its initial shape or come to breaking instability (if steepness is large). This phenomenon is a subject of forthcoming paper.

Remarkably, conformal mapping made possible a reproduction of the essential stages of the breaking process when the surface ceases to be a single-valued function (Chalikov and Sheinin, 2005). This initial stage of wave breaking exhibits a sharp jet originating from a wave crest. The integration in this case is always terminated; but due to high accuracy of the scheme, however, the numerical and physical instabilities follow each other very closely. Two ways of treating breaking were exploited. When breaking is the subject of investigation, it is simulated explicitly to the point of instability. (The last, unrealistic phase of breaking is easily eliminated from consideration of the energy conservation.) The final stage of breaking is evidently a non-potential phenomenon. This direct method is inapplicable when used for long-term simulation of the evolution of multi-mode wave fields and, for modeling of wave/wind interactions (Chalikov, 1998). For these reasons we developed a method of parameterization of breaking effects based on a high-order selective smoothing (Chalikov, Sheinin, 2005). However, in calculations considered here, we did not use this parameterization; all cases in which breaking instability took place were simply excluded from consideration.

\section{Statistical properties of wave field}

In this study, we applied the method for numerical simulation of surface waves developed in ChSh to investigation of statistical properties of nonlinear wave fields. Generally, this investigation confronts complications associated with specific wave instability - wave breaking. If initial wave energy is large, the onset of wave breaking leads inevitably to termination of calculations. Still, this instability can be eliminated with algorithms of breaking parameterization. If such algorithms are applied, however, the statistics of free waves can be distorted. For example, this smoothing algorithm eliminates the appearance of high, sharp waves.

Therefore, we first investigated the dependence of time to the onset of wave breaking on the initial conditions. The appropriate integral characteristics for fixed length scale might be the initial energy. For different length scales, this characteristic is incomplete. More appropriate is the initial steepness of surface $s$ :

$s \propto\left(\int_{0}^{M} k^{2} S(k) d k\right)^{\frac{1}{2}}$ 


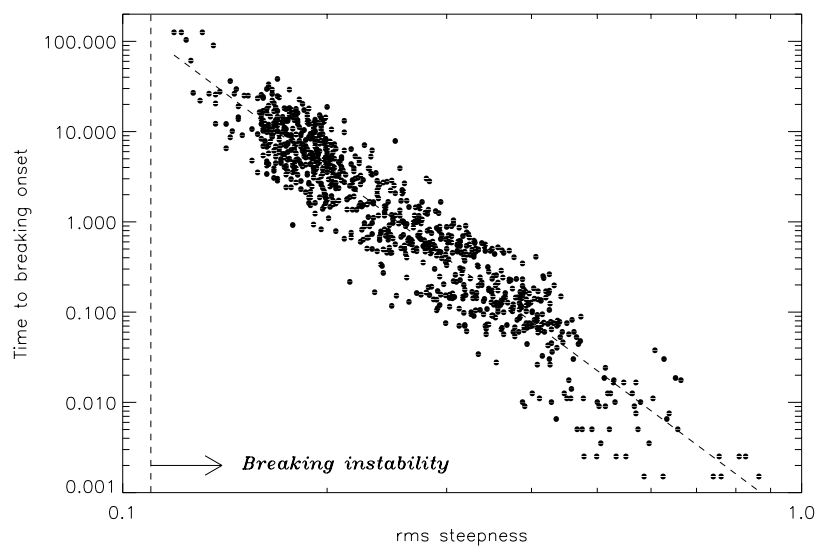

Fig. 2. Dependence of time to onset of breaking instability on rms steepness $s$. Dotted line is approximation Eq. (39)

Initial conditions were assigned as superposition of linear waves with amplitudes

$a_{k}=\left\{\begin{array}{cc}a_{0}\left(\frac{k}{k_{0}}\right)^{-m} & k_{0} \leq k \leq k_{d} . \\ 0 & \text { otherwise. }\end{array}\right.$

and random (different for each case) sets of phases. The mode with wave number $k_{0}$ has amplitude $a_{0}$, with amplitudes above $k_{0}$ decreasing as $k^{-m}$. The value of $k_{0}$ defines the resolution of the spectrum, so it cannot be too small. In most calculations, $k_{0}=10$ and $m=6$ were chosen. Further increases of $k_{0}$ did not change the results, which are described below. The statistical results were also very close when $m=5$ was chosen. Because the steepness $s$ for steep (but not yet breaking) waves decreases slightly during long-time simulations (due to tail dissipation (33)-(35)), the effective steepness for each run was obtained by averaging over time. The number of modes $M$ was 400, with grid points $N=1600$, time step $\Delta \tau=0.0025$. Calculations were performed for 70 cases

$a_{l}=0.09+0.001 \cdot l, \quad l=1,2,3 \ldots 70$

Onset of breaking was recognized when total energy started growing then exceeded its initial value by $1 \%$. This criteria specifies time of breaking onset with high accuracy, because the 4th order Runge-Kutta scheme develops instability very quickly. The onset of breaking depends not only on total nonlinearity of the initial wave field but, as well, on the initial set of phases. However, this dependence is much weaker than the dependence on steepness. To incorporate the effect of initial phases, the calculations Eq. (38) were repeated with different sets of phases. Total number of runs: 980 . The dependence of time to breaking $T_{l}$ (time $\tau$ normalized by period of peak wave $T_{p}=2 \pi / \sqrt{k_{0}}$ ) on effective steepness is demonstrated in Fig. 2. Each point was obtained by averaging over the sampling set with a total length of 160000 values (100 wave profiles).

As we show, the stability of a wave field and its existence without breaking decreases quickly with increasing the steepness. For large initial steepness of the order of 1, breaking occurs immediately and dependence on initial phases becomes much more significant. However, for effective steepness $s$ less than 0.11 the breaking was virtually absent. This statement can not be considered as an unequivocal. Still, our results confirm that breaking onset below $\mathrm{s}=0.10$ in runs up to $t=2,500$ was not observed. The dependence of $T_{k}$ on $s$ may be approximated by the formula

$T_{k}=4.6 \cdot 10^{-4} s^{-5.61}$,

Approximation (39) has a right asymptotic behavior $\left(T_{k} \rightarrow \infty\right.$ at $s \rightarrow 0$ ); it is unlikely that the specific form of this dependence is correct for values of $s$ smaller than here explored. The number of modes used in our calculations was, much larger than in calculations made by Song and Banner (2002), but a value of threshold for onset of breaking $\mathrm{s}=0.10$ is close to that in their paper.

For calculations of statistical characteristics of waves, the 10 long-term runs were performed up to 1000000 time steps (790 periods of peak wave) with initial peak steepness in the range of $a_{0} k_{0}=0.0001 \div 0.09$, corresponding to an effective steepness in the range $s=0.0001 \div 0.106$. The number of modes $M$ for the cases $1 \div 7$ was 400 , the number of grid points $N=1600$, time step was 0.0025 . For cases $8 \div 10$ (corresponding to large steepness), the tail dissipation Eqs. (33) $-(35)$ for $M=400$ was large. To reduce this effect, the number of modes for these cases was increased to $M=1000(N=4000)$, although time step and the number of steps were the same as for $\mathrm{M}=400$. The amplitudes $a_{k}$ and corresponding effective steepness are outlined in Table 1.

The wave spectra and rate of dissipation are represented in Fig. 3. The high wave-number part of spectrum fluctuates within the range of its averaged values, the amplitudes of these fluctuations grow with increasing of initial steepness and wave number. Gray areas in right part of the frames correspond to the tail dissipation function (See Eq. 35).

$D_{k}=\mu_{k}\left(z_{k}^{2}+z_{-k}^{2}\right)$

Tail dissipation is located in the high- frequency part of the spectrum. It removes the fast growing but very small modes in a vicinity of the cut wave number $M$. This dissipation is so weak that it does not influence significantly the conservation of total energy. For the cases $1 \div 7$, the energy decreases during a period of integration in 10th decimal digits; for the steepest initial conditions (case 10) the energy decreased at $10^{-3} \%$. Generally, the accuracy of conservation the total energy rises quickly with increasing spectral resolution (and shifting of the tail dissipation into the higher wave numbers). The straight line in Fig. 3 corresponds to the spectrum $S \propto k^{-6}$. Clearly, the averaged spectrum adheres closely to this dependence. Note that this property is independent of initial conditions (37). We assigned the value $m=6 \mathrm{sim}$ ply because it corresponds to the spectrum developing in the process of integration. The initial spectrum may be assigned arbitrarily providing the rms steepness lies beyond critical values $s=0.11$. The same spectrum as in Fig. 3 was obtained 
Table 1. Parameters of numerical experiments.

\begin{tabular}{lllllllllll}
\hline$\#$ & 1 & 2 & 3 & 4 & 5 & 6 & 7 & 8 & 9 & 10 \\
\hline$a_{0}$ & 0.00001 & 0.001 & 0.002 & 0.003 & 0.004 & 0.005 & 0.006 & 0.007 & 0.008 & 0.009 \\
$\mathrm{~s}$ & 0.0001 & 0.013 & 0.026 & 0.029 & 0.039 & 0.052 & 0.079 & 0.089 & 0.099 & 0.106 \\
$M$ & 400 & 400 & 400 & 400 & 400 & 400 & 400 & 1000 & 1000 & 1000 \\
\hline
\end{tabular}
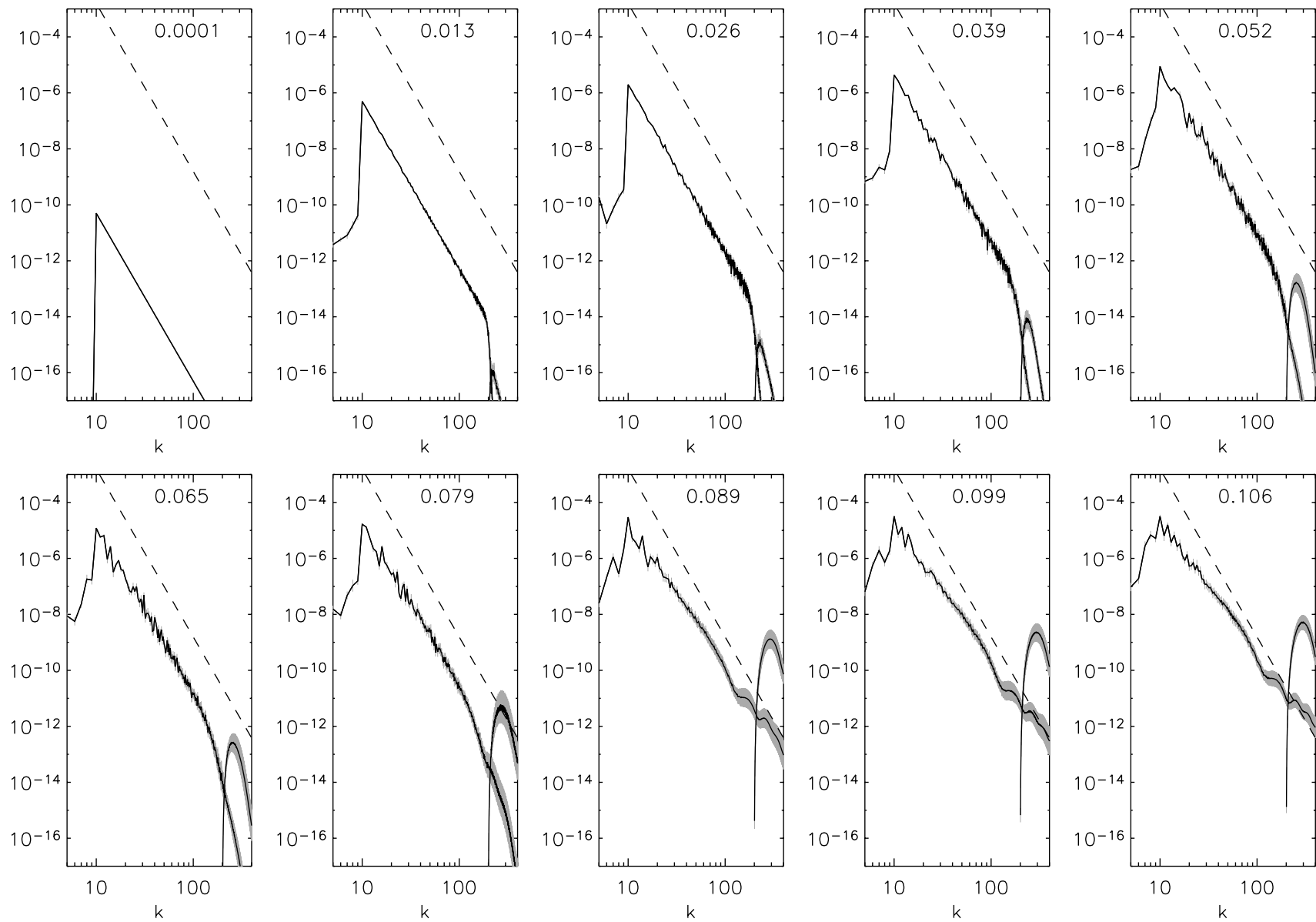

Fig. 3. Averaged for $t=2500$ (790 wave-peak periods) wave spectra, initially assigned by Eq. (37). The curves in the right side of each panel are rates of dissipation of potential energy (Eq. (40)). Straight line corresponds to $S=k^{-6}$ function. The grey vertical bars are the scatter of the rms of the spectra, and the rate of dissipation.

for $m=5$ and $k_{d}=10$. As will be shown below, fast adjustment of the spectrum to its quasi-equilibrium shape is due to a strong nonlinearity, which makes the time scales for the individual high wave-number components very short.

Fast modification of the wave field due to nonlinearity is clearly seen in Fig. 4, in which the long-time evolution of the amplitudes of the first six components (with the wave numbers $k=10 \div 15$ ) for different rms steepness are given. Only for extremely flat waves with rms steepness of the order of $10^{-4}$, the wave amplitudes remain constant over a long time. For the rms steepness $a k=0.013$, the 15th amplitude fluctuates significantly. With increasing steepness these fluctuations spread between all modes in a spectral peak, further the amplitudes of fluctuations grow with increasing steepness. A portion of these fluctuations may be attributed to exchange between potential and kinetic energy. However, because the fluctuations are much larger than sum of kinetic and potential energy for each component, the explanation of such behavior musr consider the fast energy exchange between wave modes. Strong variation of the energy of wave components occurs at very strict conservation of total energy (we recall that cases with development of breaking instability were excluded). Qualitatively, this process is similar to convergence of energy in physical space studied by Song and Banner (2002). 

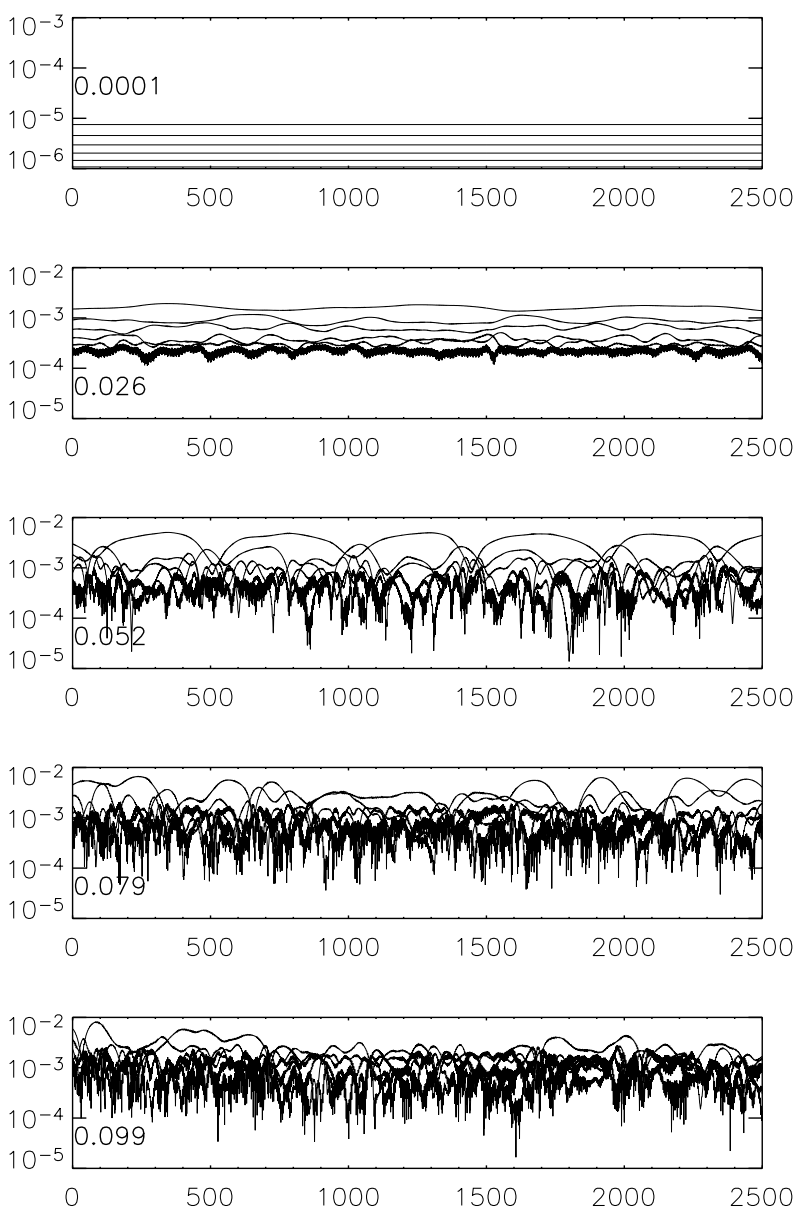
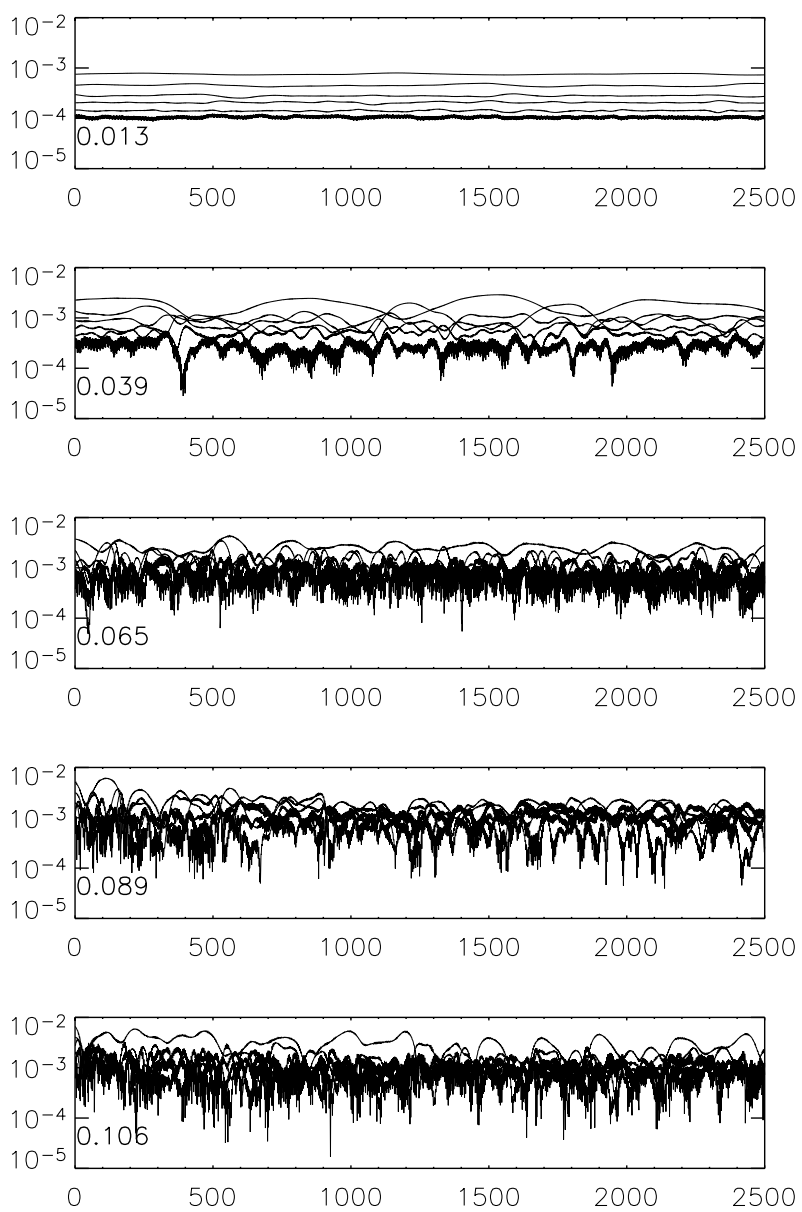

Fig. 4. Evolution of five consequent amplitudes starting from peak wave number $k=10$ for different steepness during 790 peak periods.

It is useful to consider the time scale $T_{l}$, characterizing the typical "life time" of the wave components

$T_{k}=E_{p} \frac{k^{\frac{1}{2}}}{2 \pi}\left(\overline{\left(\frac{\partial E_{p}}{\partial t}\right)^{2}}\right)^{-\frac{1}{2}}$

where $E_{p}=\frac{1}{2}\left(h_{k}^{2}+h_{-k}^{2}\right)$ is the potential energy of $k$-th wave component in the Cartesian coordinate system. Time scale $T_{k}$ is normalized by the period of linear wave component $2 \pi k^{1 / 2}$. Dependences of $T_{k}$ on the wave numbers for different rms steepness are given in Fig. 5, together with their scatter. Again, all wave components are stable for very small steepness only: their amplitudes remains constant over hundreds, even a thousand of periods. As frequency increases, time scale $T_{k}$ near the wave peak decreases up to 100 for $s=0.026$, then up to 10 for $\mathrm{s}=0.106$. For $\mathrm{k}>20$, the time scales are very small (of the order of one period of linear wave with the same wave number) for all steepness considered. An exception is the case with $s=10^{-4}$. The spectral energy of wave components for $k=20$ is smaller at three decimal orders than in a wave peak. Evidently, such transient fluctuations cannot be attributed to the free surface waves, rather to wave turbulence (Zakharov, et al., 2004; Dyachenko et al., 2004).
This result becomes more evident if one considers the phase velocities of waves. One way to calculate an instantaneous value of the phase velocity of the $\mathrm{k}$-th wave component can be done with the relation

$c_{k}=\frac{z_{-k} \frac{\partial z_{k}}{\partial \tau}-z_{k} \frac{\partial z_{-k}}{\partial \tau}}{k\left(z_{k}^{2}+z_{-k}^{2}\right)}$,

where the derivatives on time are just right side of equation (17) written for Fourier components of $z$. It is convenient to use Eq. (42) for calculations by the rms method

$\overline{c_{k}}=\frac{\overline{A D}}{\overline{D^{2}}}$,

( $\overline{c_{k}}$ is a mean phase velocity, $\mathrm{A}$ is the numerator and $\mathrm{D}$ is the denominator in (42)), and rms of phase velocity $c_{k}$

$c_{r m s}=\left(\overline{\left(c_{k}-\overline{c_{k}}\right)^{2}}\right)^{\frac{1}{2}}$

The dependencies of the phase velocities and their rms on wave numbers for different steepness are shown in Fig. 6. Each value of the phase velocity is calculated over an ensemble of 2500 wave records. Each record has a length $\mathrm{N}=1,600$ (for cases $1 \div 7$ ) or $\mathrm{N}=4,000$ (for cases $8-10$ ). A 

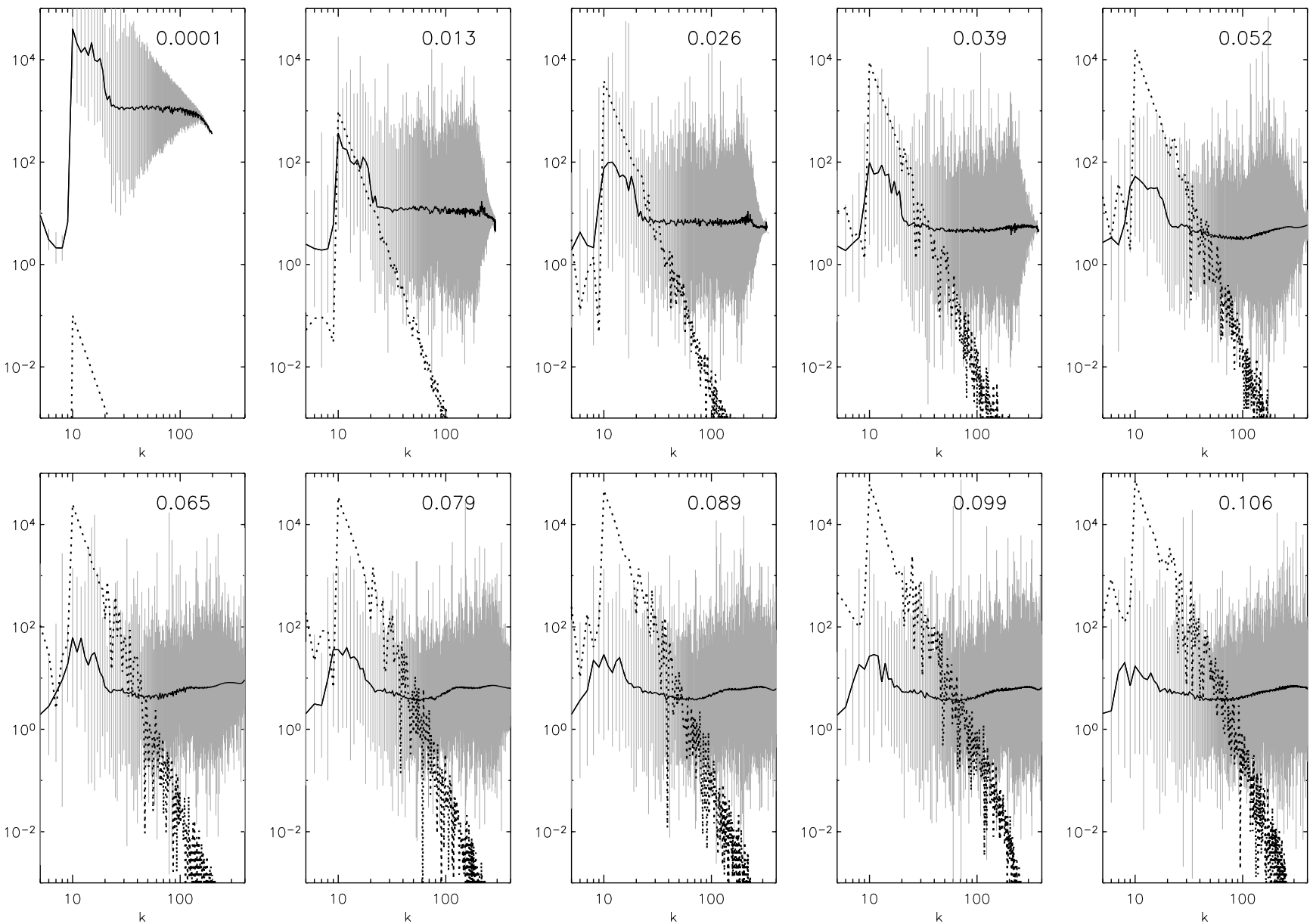

Fig. 5. "Life time" of the components of the wave field $\sim T_{k}$ expressed in periods for every specific component (Eq. (41); solid lines). Grey lines indicate the scatter of $\sim T_{k}$. Dotted lines are the averaged wave spectrum. The numbers in the right upper corner are the effective steepness.

linear dispersion relation $c_{k}=k^{-1 / 2}$ is perfectly accurate only for $s=0.0001$. For waves with $s=0.013$, the fluctuations of phase velocity for $k=20$ become noticeable. Further increase of steepness increases the phase velocity of the high modes and its fluctuations grow. A simple explanation of these phenomena was given in ChSh. In fact, at each wave number several modes coexist: one a free wave, others so-called "bound waves" corresponding the additional modes attached for every steep enough wave. The calculation of phase velocity based on Eqs. (42) and (43) gives a weighted value between the velocities of free and several bound modes. A separation of bound waves and free waves was conducted in ShCh by calculation a wave number-frequency spectrum. Bound waves are attributed to different carrying waves, so some have identical wave numbers. The amplitudes of carrying waves change in time, due to nonlinearity (see Fig. 4), so a situation becomes very complicated. In order to understand how shape of wave differs from what it is assumed in linear theory, calculations of high-order moments for different steepness were performed.
The function $z(\xi)$ was transferred from conformal coordinates to function $\eta(x)$ in Cartesian coordinates, using 4th order periodic polynomial spline (providing an accuracy of the order of $10^{-11}$ ) and then recorded for processing. Every hundred such records were linked in single set with length of $L=160,000$, which then was used for calculation of statistical characteristics: mean $\bar{\eta}$; variance $V$; skewness $S$; and curtosis $K$ :

$$
\begin{gathered}
\bar{\eta}=\frac{1}{L} \sum_{j=0}^{L-1} \eta_{j}, V=\frac{1}{L-1} \sum_{j=0}^{L-1}\left(\eta_{j}-\bar{\eta}\right)^{2}, S=\frac{1}{L} \sum_{j=0}^{L-1}\left(\frac{\eta_{j}-\bar{\eta}}{\sqrt{V}}\right)^{3}, \\
K=\frac{1}{L} \sum_{j=0}^{L-1}\left(\frac{\eta_{j}-\bar{\eta}}{\sqrt{V}}\right)^{4}-3,
\end{gathered}
$$

The value of $\bar{\eta}$ is very small and remains constant (strict conservation of volume, see $\mathrm{ChSh}$ ), and $V$ is a doubled potential energy. When a wave field is a superposition of a large number of harmonic waves, both the skewness and curtosis are equal to zero. Skewness $S$ characterizes the asymmetry of the distribution of probability. If the positive values of $\eta$ are 

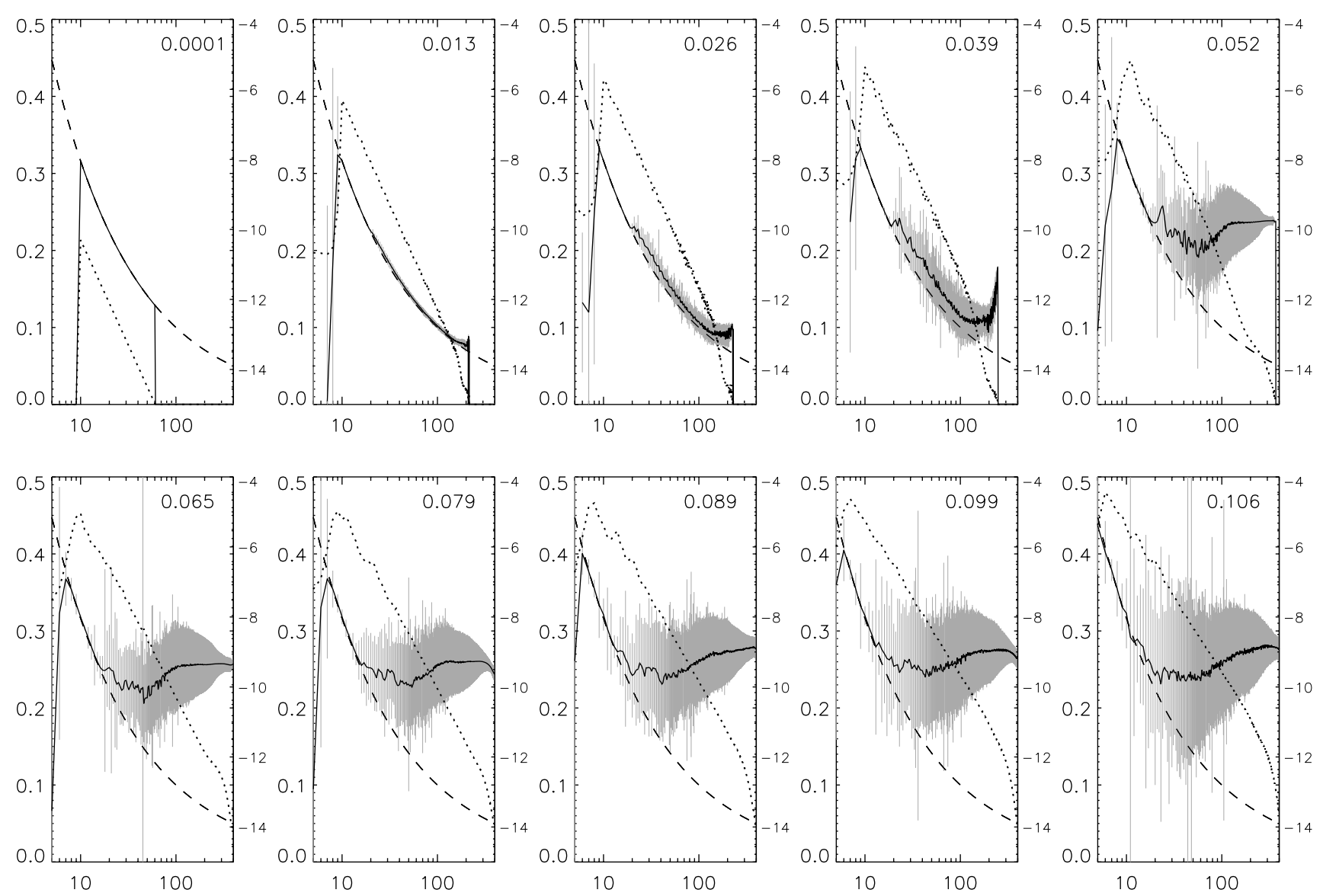

Fig. 6. Phase velocities of wave components as function of wave number (see Eq. (43)). Dashed line is a linear dispersion relation. Grey vertical bars correspond to rms of phase velocities (Eq. (44)). Dotted line is a wave spectrum (right axis).

larger than the negative values, then $\mathrm{S}>0$. Curtosis is positive if the crests are sharper and the troughs are smoother than in a case of linear waves. As seen in Fig. 7, the skewness is very close to zero for $\mathrm{s}=0.001$ only; with increasing nonlinearity it grows rapidly and reaches as large a value as $\mathrm{S}=0.35$ - corresponding to significant exceeding of heights of crests over depth of troughs. Qualitatively, these properties are supported by data on curtosis (Fig. 8) growing with increasing nonlinearity, and confirming that wave crests become sharper and troughs more gentle for steep waves.

Data on skewness and curtosis (Figs. 7 and 8), observations of wave height records as well as the results of simulations based on principal wave equations (as in Fig. 12) always exhibit the fundamental properties of nonlinear wave fields: the waves tend to be sharper and higher than harmonic waves.

A question arise: do the Stokes waves posses any practical utility or they are simply example of an analytical solution for stationary gravity waves - waves so unstable that they never exist? Does a routine Fourier presentation used in most of theoretical and experimental investigations miss the nonlinear nature of steep waves?

Attempting to answer, a presentation of nonlinear wave field as superposition of Stokes waves was tried. Naturally the functions $S_{k}$ corresponding to the Stokes waves are not orthogonal, so calculation of coefficients in expansion

$h(x)=\sum_{o}^{M} \sigma_{k} S_{k}\left(\sigma_{k}\right)$

converts to minimization problem. Because the shape of Stokes waves $S_{k}$ depends on its amplitude $\sigma_{k}$, this is a nonlinear problem - complicated but still resolvable. However, a more elegant solution was found.

Let us to consider the conformal coordinates for upper domain $z>\eta$.

$$
\begin{aligned}
& x=\xi_{a}-\sum_{-M \leq k \leq M, k \neq 0} \nu_{-k}(\tau) \frac{\cosh k\left(\left[H_{a}\right]-\varsigma_{a}\right)}{\sinh k H} \vartheta_{k}\left(\xi_{a}\right) \\
& z=\zeta_{a}+\sum_{-M \leq k \leq M, k \neq 0} \nu_{-k}(\tau) \frac{\sinh k\left(\left[H_{a}\right]-\zeta_{a}\right)}{\sinh k H} \vartheta_{k}\left(\xi_{a}\right)
\end{aligned}
$$

where $v_{k}$ are the Fourier coefficients of presentation of the interface, $\xi_{a}, \zeta_{a}$ are the conformal coordinates in upper domain. The transformations Eqs. (9), (10), and (47) have somewhat opposite behavior: where the Jacobean in a lower coordinates is large (in the crests), in upper coordinates it 


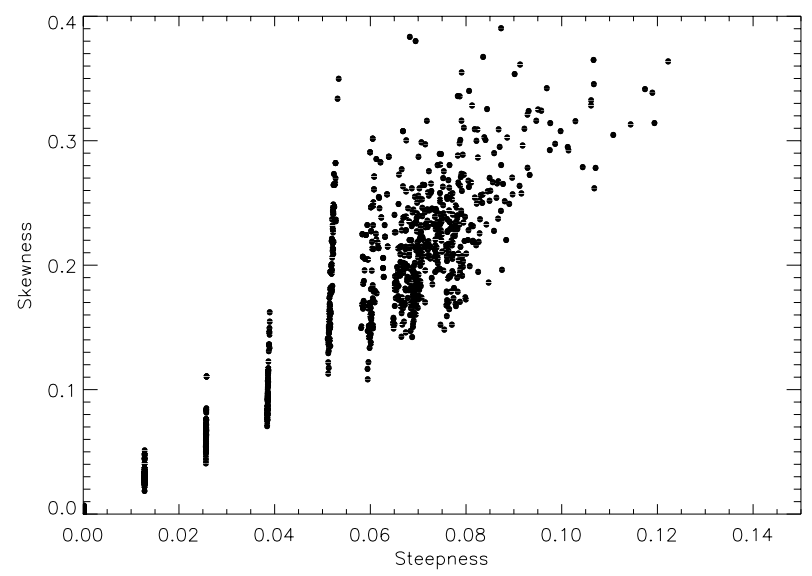

Fig. 7. Skewness of wave field as a function of the effective steepness (Eq. (45) third formula). Each point is obtained by averaging over a sampling set with total length of 160000 values (100 wave profiles).

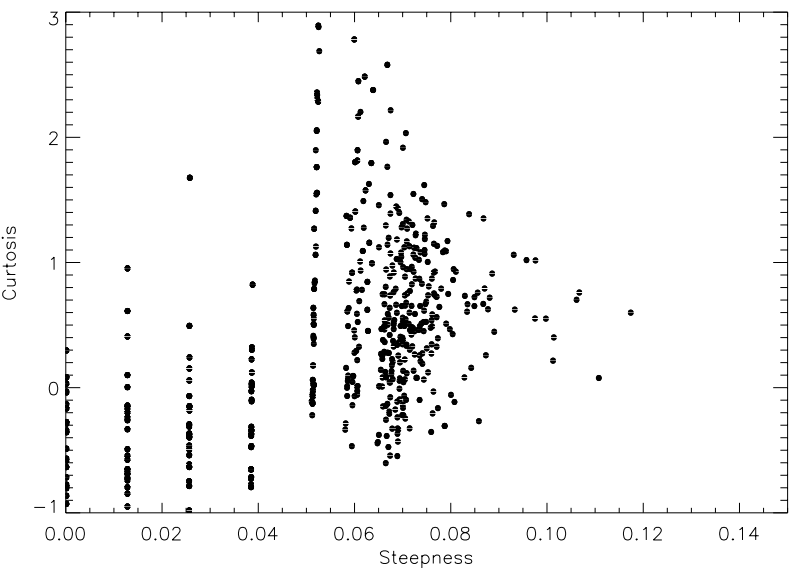

Fig. 8. The same as in Fig. 7, but for Curtosis of wave field (Eq. (45), forth formula).

is small. The opposite applies for the troughs. The system of coordinates Eq. (47) is used now for modeling of turbulent flow above waves and wind-wave interactions (Chalikov, 1998). In this paper, however, the first formula of Eq. (47) is used for introducing dependence $\xi_{a}(\xi)$ for infinite height $H_{a}$. An advantage of this transformation is that for the same accuracy of approximation the sharp waves in the lower coordinate need more of modes than in the upper coordinates. The number of modes for the approximation with the same accuracy in Cartesian coordinates lies somewhere between. This statement is illustrated in Fig. 9, representing the spectrum of Stokes waves with steepness $a k=0.42$ and $a k=0.43$ (for infinite depths) calculated in the lower coordinates with the method ChSh. These solutions were transferred in the Cartesian and upper coordinates with an accuracy of the order of $10^{-11}$. The convergence of Fourier expansion is fastest in the upper coordinates, and lowest in a lower coordinates. For $k=10$, the value of Fourier mode in upper coordinates is two

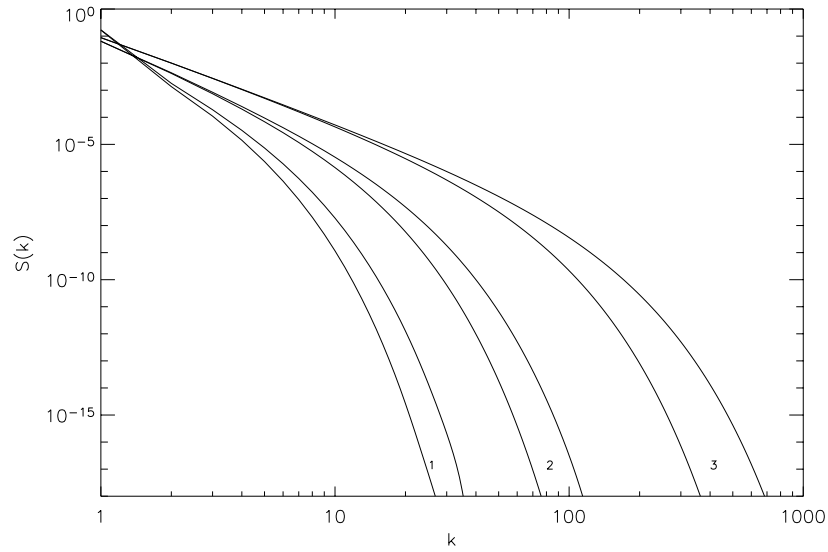

Fig. 9. Spectra of Stokes waves: (1) - in lower coordinates; (2) - in Cartesian coordinates; (3) - in upper coordinate. Thin lines correspond to $\mathrm{ak}=0.42$, thick lines to $\mathrm{ak}=0.43$.

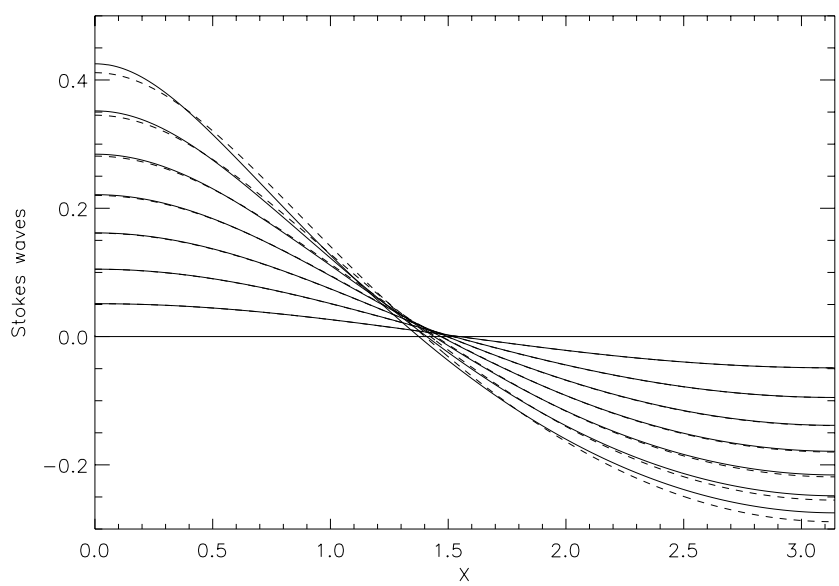

Fig. 10. Profiles of Stokes waves in Cartesian coordinates for $\mathrm{ak}=0.05,0.10,0.15,0.20,0.25,0.30,0.35(k=1$, solid lines $)$ Dashed curves correspond to $a \cos (k \theta)$ (see Eqs. (47)).

decimal orders smaller than in Cartesian coordinates and by three decimal orders than in lower coordinates.

It is reasonable to consider what shape obtains the single mode $a \cos \left(\xi_{a}\right)$ in upper coordinate transferred to Cartesian coordinate. The results for $\mathrm{a}=0.05,0.10,0.15,0.20,0.25$, 0.30 and 0.35 are represented by a dashed line in Fig. 10 . Solid lines represent the exact Stokes wave profiles calculated in lower coordinates (with method ChSh) and transferred in Cartesian coordinates. As shown, the shape of Stokes wave for $a \leq 0.25$ coincides with the shape of a single mode in upper coordinates. For $a=0.30$ and $a=0.35$, the difference between the two curves is noticeable but yet small. Typically, the steepness of sea waves rarely exceeds the value $a k=0.30$. So, a Fourier presentation of wave surfaces in the upper coordinate may be considered with reasonable accuracy as expansion over Stokes waves, which are nearly orthogonal in the upper coordinate system. These modes are orthogonal in Cartesian coordinate system with 

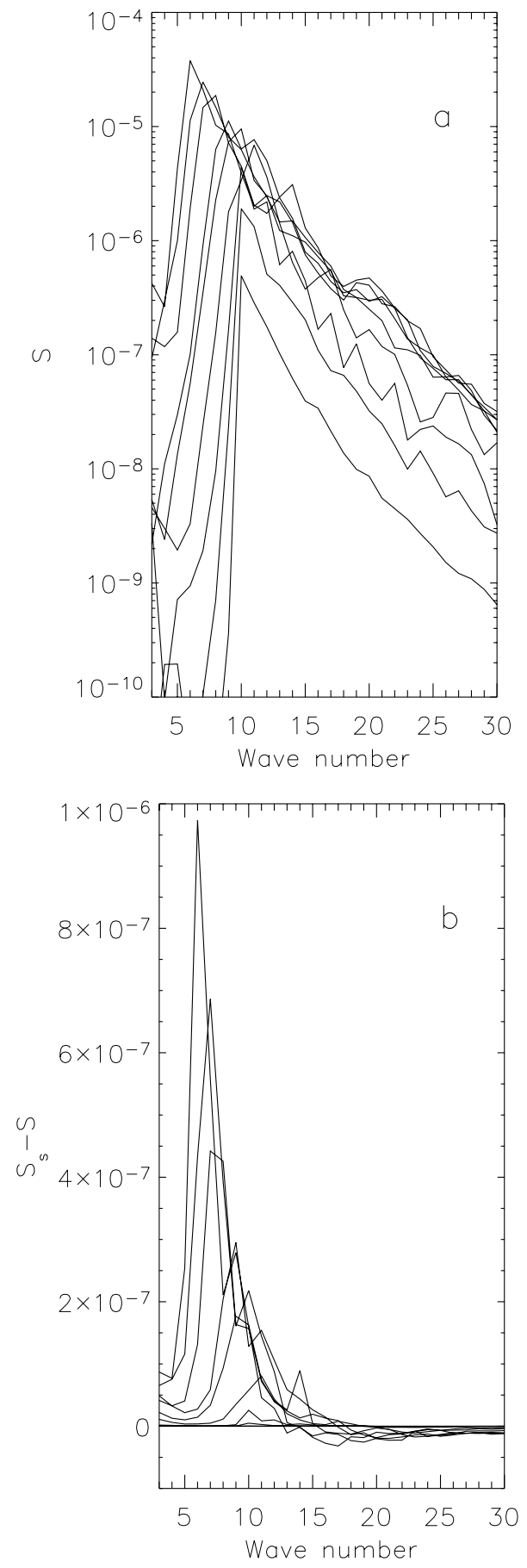

Fig. 11. Upper panel - the same as in Fig. 3 spectra in linear kscale; Lower panel - difference between spectra and "spectrum" over Stokes waves.

weights corresponding to the inverse Jacobian of conformal mapping to upper coordinates. The left panel in Fig. 11 represents the averaged wave spectra calculated in Cartesian coordinates for ten cases (Table 1), and the bottom panel is the difference between averaged wave "spectrum" in upper coordinates and spectrum in Cartesian coordinates. Plainly, the difference at low wave numbers is large and positive, in high wave numbers negative. This means that representation of a surface as a superposition of Stokes waves is more compact than routine representation as superposition of linear waves. It is well known that sea waves usually have sharp crests and gentle roughs. All quasi-linear theories ignore this evident property of actual wave fields. This reality explains, qualitatively, the increasing the skewness and curtosis for steep waves shown in Figs. 7 and 8.

Visual observations of long-term evolution of wave surface shows that steep enough wave fields has quasi-periodic behavior: a period of more or less smooth waves followed by period when large waves becoming sharper (the same effect as observed by Song and Banner, 2002). In our calculations, the length of domain was equal to ten lengths of peak wave. During the period of sharpening, the several waves may become sharper simultaneously; more often, however, only one wave become sharper. In Fig. 12 (panel 1), the evolution of kinetic and potential energies for $\mathrm{s}=0.089$ is given. Both energies fluctuate with amplitude up to $10 \%$, their sum remains constant within many decimal digits. In panel 2 , the top curve represents the evolution of the maximum wave height defined over whole period for 2,500 wave profiles separated by interval $\Delta t=1$; the bottom curve depicts evolution of the minimum value of the second derivative $\partial^{2} \eta / d x^{2}$ for the same set. The intervals of increasing wave height always coincide with minimums for the second derivatives, corresponding to sharpening of crests. In panel 3, the "sharpest" wave profile for time $t=815$ (corresponding the minimum of $\partial^{2} \eta / d x^{2}$ ) is drawn (dotted curve); solid line is the smoothest wave profile (minimum of absolute value of $\partial^{2} \eta / d x^{2}$ ). Both profiles are equally smooth, except that the first has single high peak. The wave-number spectra of these profiles are given in panel 4 . The spectrum corresponding to the first case has much larger high wave-number values. All these components were needed for correct approximation of a ingle sharp peak in a domain. In sum, for large number of cases for developed wave fields a considerable part of high frequency does not correspond to actual waves, but artifact, created by attempting a linear presentation of a strongly nonlinear process. In reality, a concentration of energy occurs in a physical space, rendering its Fourier presentation meaningless. This conclusion strongly supports the results of Song and Banner (2002). This also explains, why formally calculated time scales (Eq. 41 and Fig. 5) for high frequency waves are so small.

The nonlinear properties of waves create specific integral probability distribution. In Fig. 13, the probability of troughto-crest heights $H_{t c}$ (normalized by significant wave height $H_{s}$ ) for large waves is displayed. Trough-to-crest height of large waves was defined as maximum minus minimum wave heights in a moving window with width equal to $1.5 L_{p}$ for each of 2500 wave profiles. The probability of large waves increases significantly with increasing steepness. For large waves, the dependence of maximum wave height on effective steepness is not monotonic. one reason is that very large waves are rare, so the calculation of accurate statistics requires a larger dataset. 

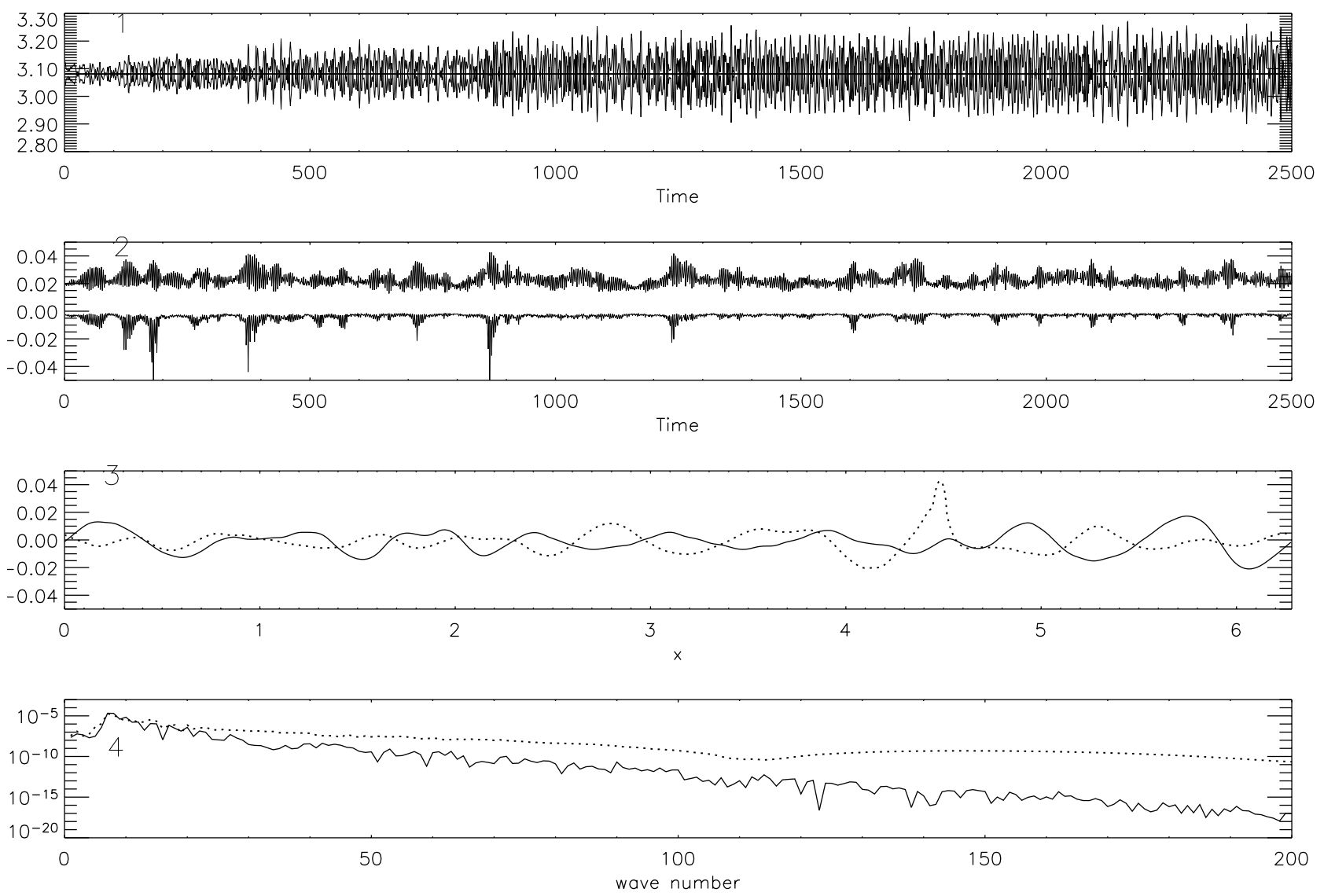

Fig. 12. (1) - The evolution of kinetic and potential energy (thin lines). Their sum (thick line) remains constant throughout simulation. (2) - The top curve represents evolution of the maximum wave height defined over an entire period for 2500 wave profiles separated by interval $\Delta t=1$; bottom curve - evolution of minimum value of the second derivative $\partial^{2} \eta / d x^{2}$ for the same data set. (3) - the "sharpest" wave profile for time $\mathrm{t}=815$, corresponding to the minimum of $\partial^{2} \eta / d x^{2}$ (dotted curve); the "smoothest" wave profile for time $=2,224$, corresponding to the maximum (minimum of absolute value) of $\partial^{2} \eta / d x^{2}$ (solid line). Equation (4) - the wave spectrum corresponding the "sharpest" wave profile (dotted line) and the "smoothest" wave profile (solid line).

\section{Discussion and conclusions}

In this study, we applied the method for numerical simulation of periodic surface waves, developed in ChSh, to longrange simulation of multi-mode wave fields. The principal equations are the standard equations of hydrodynamics for potential flow with a free surface. The method is based on a nonstationary conformal transformation that maps the original domain (which may be of finite or infinite depth) onto a domain with a fixed rectilinear upper boundary. For the stationary problem, the method is identical with the classic complex variable method. In the transformed coordinates, the solution to the Laplace equation for the velocity potential is represented with Fourier series. This eliminates the need for finite-difference approximation of spatial derivatives and reduces the problem to 1-D. Numerical solution of the initialvalue problem for the transformed system thus becomes a straightforward task, and is reduced to time integration of two simple evolutionary equations for the surface velocity potential and surface height. These variables are represented by their Fourier expansions with time-dependent coefficients.
The Fourier transform method used for calculation of the nonlinear terms provides excellent computational efficiency.

This model has important advantages: (1) comparison with an exact solution shows that the scheme demonstrates extremely high accuracy (see Fig. 1); (2) it preserves precisely the integral invariants; (3) it is very efficient: performance decreases as $\operatorname{Mlog}(\mathrm{M})$ ( $M$ is the number of modes); (4) the scheme demonstrates stability over millions of time steps (thousands of the periods of dominant wave); (5) the speed of calculations is fast enough to perform any reasonable numerical experiments on a personal computer. This model is able to reproduce a nonlinear concentration of energy in physical space resulting in the appearance of large and steep (freak) waves and an approaching to wave breaking. Remarkable, the initial equations (not necessarily potential, but adiabatic) preserve similarity over the length scale (outside of capillary range). Accordingly, wave model - by simple scaling - may be applied to any scales. The breaking waves and the freak waves therefore have the same nature, the breaking waves appearing as the freak waves in the Lilliputian country. 


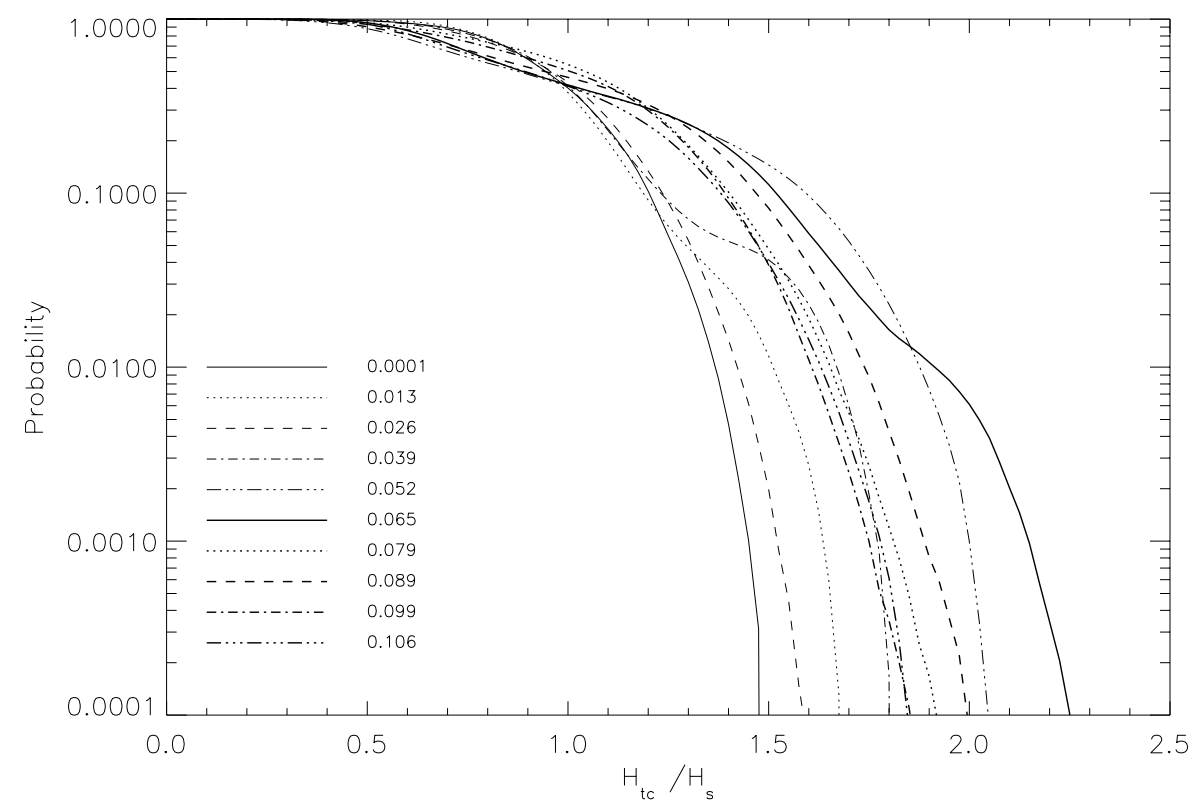

Fig. 13. Integral probability of the trough-to-crest wave height, normalized by significant wave height.

A consideration of time scales for the multi-mode wave field with initially random phases confirms that lowfrequency waves preserve their individuality, though their "life time" decreases with increasing steepness. The total energy of each mode always fluctuates, because of quasiperiodic energy exchanges between wave components. For high frequencies "life time" is an order of one period. These disturbances cannot be attributed to waves, rather to "wave turbulence". Applicability of the 1-D approach and potential assumption to high frequency waves is very questionable. This approach obviously cannot simulate properly the processes where irreversible 2-D nonlinear interactions are of the essence. However, based on results of this work, the important conclusion follows for 2-D waves also. Of course, all nonlinear effects in the 2-D case should be pronounced clearer because of an infinitely larger number of interacting modes and physically because of a complexity of the orbital velocity field. However, for wind-wave interaction problems, 1-D wave model is acceptable because it reproduces a broad spectrum of waves and surface disturbances; these, in turn, generate rich statistics of nonlinear fluctuations in an air flows above waves.

The model developed may be applied to a broad range of situations in which the 1-D approximation is acceptable. Fortunately, many wave phenomena are largely controlled by strong nonlinear interactions which are relatively fast and for which the 1-D approximation is often adequate. Formation of extreme waves is one such phenomenon. As yet, model simulations of vary large waves are far from academic interest only. It has long been known that nonlinear redistribution of energy can result in the abrupt emergence of very large and steep waves. commonly known as freak or rogue waves. Amplitude and phase modulations create especially favorable conditions for their formation.
It is well known that in a real wave field the dominate waves have more or less sharp crests and gentle troughs. When a routine Fourier presentation is used, for approximation of such waves the additional modes are needed which are sometimes called "bound waves". This unfortunate expression obscures the essence of the phenomena; real waves are single nonlinear modes which preserve their individuality over time. For reasons not yet understood, the shape of such waves is close to harmonic modes in the "upper" coordinate system. These modes form an orthogonal basis in the "upper" coordinate system and are also orthogonal in the Cartesian coordinate system with the weights equal to the inverse Jacobian of transformation to "upper" coordinate. Remarkable, that Fourier expansion for stationary solutions for potential waves in the "upper" coordinate system (Stokes waves) converges faster than in Cartesian coordinate system. For actual wave field with moderate steepness, the superposition of Fourier modes in "upper" coordinate is very close to superposition of Stokes waves in Cartesian coordinates. Probably, this fast convergence for Stokes solution for potential waves in upper coordinate is a rational result. Nonlinear transformation "absorbs" somehow the nonlinearity. And the single mode in upper coordinate (contrary to that in usual coordinates) well approximates the solution of strongly nonlinear equations. The derivation of equations in "upper" coordinates is tricky, because "there is no fluid". However, this idea may be not fully impractical because potential waves equations are essentially "surface" equations. As well, these coordinates correspond the case of two-layer flow with the interface with density of the upper liquid approaching zero. Important additional advantage of the "upper" coordinate system is that singularities of interface may disappear or at least can weaken. 
The most important application of the scheme developed here is the coupled modeling of waves and wave boundary layer (see Chalikov, 1998). It can not be proved that multimode wave surface interacts with atmosphere as a set of independent waves, and that the integral result can be obtained by simple superposition of monochromatic cases. It is well known that even a single wave can produce a broad spectrum of pressure fluctuations which affect the flow. Atmospheric response to a strongly nonstationary wave field is also essentially nonstationary. The structure of nonstationary flow (e.g. a distribution of surface pressure) is different of stationary. First attempt to take into account these nonlinearity and group effects was done with our finite-difference model (see review Chalikov, 1986), in which wave surface was assigned as superposition of running linear waves with different frequencies. That approach is much closer to reality than approach based on stationary models, because it allows reproducing a group structure of waves and its nonlinear consequences. However, although better (and more complicated) than the monochromatic stationary approach, that model proved imperfect as well, because the specifics of real wave shapes and nonlinear group structure were not represented.

Majority of works devoted to wind-wave interaction consider a single-mode surface. This approach is oversimplified - to be used for simple qualitative analysis only. Linear approaches are wholly inapplicable for such complicated issues as type of closure scheme to apply to a full nonlinear problem. Many works use a nonlinear approach based on Reynolds equations, with most considering the stationary flow above monochromatic waves (e.g. the simulations of Mastenbroek et al., 1996, Meirlink and Makin, 2000 - both based on model, created by Chalikov, 1976). This approach is not full, because even small disturbances of obstacles (like sharpening of crest) produce dramatic changes in pressure field and form drag (this effect is well known in engineering fluid mechanics). Simple group effect generates high and steep waves (in physical space) with deep minimum of pressure behind their crests. Nonlinearity enhances the effect of sharpening, thereby, strongly increasing the pressure anomalies. The averaged wave drag and energy exchange result from an ensemble effect of what are essentially nonstationary fluctuations of pressure and surface stresses. These processes are absent in routine monochromatic stationary models.

The main disadvantage of 1-D approach is a weak nonlinearity resulting in the formation of a fast decreasing spectrum $\left(S \propto k^{-6}\right)$. Unfortunately, the 1-D model cannot tolerate more saturated spectrum. 2-D waves has spectrum $S \propto k^{-3 / 2}$ (Dyachenko et al., 2004).

Still, the prime conclusions from this work, as to the inapplicability of a linear dispersive relation and the transient character of high-frequency waves remain valid for 2-D waves as well.
Acknowledgements. The authors wish to thank W. F. Althoff, Research Associate, Smithsonian Institution, who edited the manuscript making a number of improvements, as well as unknown reviewers for their valuable comments and suggestions.

Edited by: R. H. J. Grimshaw

Reviewed by: two referees

\section{References}

Baker, G. R., Meiron, D. I, and Orszag, S. A.: Generalized vortex methods for free-surface flow problems, J. Fluid Mech., 123, 477-501, 1982.

Banner, M. L. and Tian, X.: Energy and momentum growth rates in breaking water waves, Phys. Rev. Lett., 77, 2953-2956, 1996.

Banner, M. L. and Tian, X.: On the determination of the onset of breaking for modulating surface gravity waves, J. Fluid Mech., 367, 107-137, 1998

Belcher, S. and Hunt, J. C. R.: Turbulent shear flow over hills and waves, Ann. Rev. Fluid. Mech. 30, 507-538, 1998.

Benjamin, T. B. and Feir, J. E.: The disintegration of wavetrains in deep water, J. Fluid. Mech., 27, 417-430, 1967.

Bredmose, H., Brocchini, M., Peregine, D. H., and Tsais, L.: Experimental investigation and numerical modeling of steep forced water waves, J. Fluid Mech., 490, 217-249, 2003.

Chalikov, D.: The numerical simulation of wind-wave interaction, J. Fluid Mech., 87, 551-582, 1978.

Chalikov, D. V.: Numerical simulation of the boundary layer above waves, Bound. Layer Met., 1986, 34, 63-98, 1986.

Chalikov, D.: Interactive modeling of surface waves and boundary layer. Ocean Wave Measurements and Analysis, ASCE, Proceeding oh the third Intern. Symp. WAVES 97, 1525-1540, 1998

Chalikov, D. V. and Liberman, Yu.: Integration of primitive equations for potential waves, Izv. Sov. Atm. Ocean Phys., 27, 42-47, 1991.

Chalikov, D. and Sheinin, D.: Numerical modeling of surface waves based on principal equations of potential wave dynamics, Technical Note, NOAA/NCEP/OMB, 54, 1996.

Chalikov, D. and Sheinin, D.: Direct Modeling of One-dimensional Nonlinear Potential Waves, Nonlinear Ocean Waves, edited by: Perrie, W., Advances in Fluid Mechanics, 17, 207-258, 1998.

Chalikov, D. and Sheinin, D.: Modeling of Extreme Waves Based on Equations of Potential Flow with a Free Surface, Journ. Comp. Phys., in press, 2005.

Craig, W. and Sulem, C.: Numerical Simulation of Gravity Waves, Journal of Comp. Phys., 108, 73-83, 1993.

Crapper, G. D.: An exact solution for progressive capillary waves of arbitrary amplitude, Journal of Fluid Mech., 96, 417-445, 1957.

Crapper, G. D.: Introduction to Water Waves, John Wiley, Chichester, 224, 1984

Dimas, A. A. and Triantafyllou, G. S.: Nonlinear interaction of shear flow with a free surface, J. Fluid Mech., 260, 211-246, 1994.

Dold, J. W. and Peregrine, D. H.: A efficient boundary-integral method for steep unsteady water waves, in: Numerical Methods for Fluid Dynamics, edited by: Morton, K. W. and Baines, M. J., Oxford University Press, 1986.

Dold, J. W.: An Efficient Surface-Integral Algorithm Applied to Unsteady Gravity Waves, Journal of Comp. Phys., 103, 90-115, 1992. 
Dommermuth, D. G., Yue, D. K. P., Rapp, R. J., Chan, F. S., and Melville, W. K.: Deep waterbreaking waves; a comparison between potential theory and experiments, J. Fluid Mech., 89, 432442, 1998

Dommermuth, D. G.: The laminar interactions of a pair of vortex tubes with a free sur-face, J. Fluid Mech., 246, 91-115, 1993.

Dommermuth, D. G. and Yue, D. K. P.: A high-order spectral method for the study of nonlinear gravity waves, J. Fluid Mech., 184, 267-288, 1987.

Donelan, M.: Air-Sea Interaction,edited by: LeMehaute, B. and Hanes, C. M., John Wiley and Sons, New York, 9, 239-292, 1990.

Drennan, W. M., Hui, W. H., and Tenti, G.: Accurate calculation of Stokes wave near breaking, Continuum Mechanics and its applications, edited by: Graham, C. and Malik, S. K., Hemisphere Publishing, 1988.

Dyachenko, A. I. and Zakharov, V. E.: Is free surface hydrodynamics an integrable system?, Phys. Lett., 190 , (2), 144-148, 1994.

Dyachenko, A. I., Kuznetsov, E. A., Spector, M. D., and Zakharov, V. E.: Analytical description of the free surface dynamics of an ideal fluid (canonical formalism and conformal mapping), Phys. Lett., A 221 (1-2), 73-79, 1996.

Dyachenko, A. I., Korotkevich, A. O., and Zakharov, V. E.: Weak Turbulent Kolmogorov Spectrum for Surface Gravity Waves, Phys. Rev. Lett., 92, 13 4501, 2004.

Eliassen, E. B., Machenhauer, B., and Rasmussen E.: On a numerical method for integration of the hydro-dynamical equations with a spectral representation of the horiszontal fields, Report 2, Institute for Teoretisk. Meteorologi, Kobenhavens Universitet, Copenhagen, 1970.

Farmer, J, Martinelli, L., and Jameson, A.: A Fourier method for solving nonlinear water-wave problems: application to solitarywave interactions, J. Fluid Mech., 118, 411-443, 1993.

Fenton, J. D and Rienecker, M. M.: A Fourier method for solving nonlinear water-wave problems: application to solitary-wave inter-actions, J. Fluid Mech., 118, 411-43, 1982.

Floryan, J. M. and Rasmussen H.: Numeri-cal methods for viscous flows with moving boundaries, Appl. Mech. Rev.,42, 323-341, 1989.

Fornberg, B.: A numerical method for conformal mapping, SIAM, J. Sci. Comput., 1, 386-400, 1980.

Fritts, M. J, Meinhold, M. J., and von Kerczek, C. H.: The calculation of nonlinear bow waves, Proc. 17th Symp. Naval Hydrodyn., , The Hague, Netherlands, 485-497, 1988.

Geernart, G. L.: Bulk parameterization for the wind stress and heat flux, in: Surface Waves and Fluxes, Vol. 1 - Current theory, edited by: Geernart, G. and Plant, W., Kluwer Acad. Publ., Netherlands, 91-172, 1990.

Gent P. R. and Taylor P. A.: A numerical model of the air flow above water waves, J. Fluid Mech., 77, 105-128, 1976.

Harlow F. H. and Welch, E.: Numerical calculation of timedependent viscous incompressible flow of fluid with free surface, Phys. Fluids, 8, 2182-2189, 1965.

Henderson D., Peregrine, D. H., and Dold, J. W.: Unsteady water waves modulations: fully nonlinear solutions and comparison with the nonlinear Shroedinger equation, Wave Motion, 29, 341$361,1999$.

Hyman, J. M.: Numerical methods for tracking interfaces, PhysicaD., 12, 396-407, 1984.

Floryan, J. M. and Rasmussen, H.: Numerical methods for viscous flows with moving boundaries, Appl. Mech. Rev., 42, 323-341, 1989.
Hirt, C. W. and Nichols, B. D.: Volume of fluid method for the dynamics of free surface, J. Comput. Phys., 39, 201-225, 1981.

Kano, T. and Nishida, T.: Sur le ondes de surface de l'eau avec une justification mathematique des equations des ondes en eau peu profonde,J. Math, Kyoto Univ. (JMKYAZ), 19-2, 335-370, 1979.

Longuet-Higgins, M. S. and Cokelet, E. D.: The deformation of steep surface waves on water, I. A numerical method of computation, Proc. R. Soc. Lond., 350, 1-26, 1976.

Longuett-Higgins, M. S.: The crest instability of steep gravity waves, or how do short waves break? in: The Air-Sea Interface, Radio and Acoustic Sensing, Turbulence and Wave Dynamics, edited by: Donelan, M. A., Hui, W. H., Plant, W. J., University of Toronto Press Inc. Toronto, 1996.

Mastenbroek, C., Makin, V. K., Garat, M. H., and Giovanangeli, J. P.: Experimental evidence of the rapid distortion of the turbulence in the air flow over water waves J. Fluid. Mech., 318, 273-302, 1996.

Meirink, J. F. and Makin, V. K.: Modelling low-Reynolds-number effects in the turbulent air flow over water waves,J. Fluid. Mech., 2000.

Meiron, D. I., Orszag, S. A., and Israeli, M.: Applications of numerical conformal mapping, J. Comp. Physics, 40, 2, 345-360, 1981.

Magnusson, A. K., Donelan, M. A., and Drennan, W. M.: On estimating extremes in an Evolving Wave Field, Coastal Engineering, 36, 147-163, 1999.

Mei, C. C.: Numerical methods in water-wave diffraction and radiation, Ann. Rev. Fluid Mech., 10, 393-416, 1978.

Miyata, H.: Finite-difference simulation of breaking waves, J. Comput. Phys., 5, 179-214, 1986.

Noh, W. F. and Woodward, P.: SLIC (simple line interface calculation), in: Lecture Notes Phys., New York Springer-Verlag, 59, 330-340, 1976.

Orszag, S. A.: Transform method for calculation of vector coupled sums. Application to the spectral form of vorticity equation, Journal of Atmos Sci, 27, 890-895, 1970.

Prosperetti, A. and Jacobs, J. W.: A Numerical Method for Potential Flow with a Free Surface, J. Comp. Phys., 51, 365-386, 1983.

Roberts, A. J.: A stable and accurate numerical method to calculate the motion of a sharp interface between fluids, IMA J. Appl. Math., 1, 293-316, 1983.

Sheinin, D. and Chalikov, D.: Numerical Investigation of Wavenumber-Frequency Spectrum for 1-D Nonlinear Waves. Office of Naval, Research (ONR) Ocean Waves Workshop, 1994, 16-18 March, University of Arizona, Tucson, AZ, Extended abstract, 1994.

Sheinin, D. and Chalikov, D.: Hydrodynamical modeling of potential surface waves, in: Problems of hydrometeorology and environment on the eve of XXI century, Proceedings of international theoretical conference, St. Petersburg, 24-25 June 1999. St. Petersburg, Hydrometeoizdat, 305-337, 2000.

Song, J.-B. and Banner, M.: On determining the onset and strength of breaking for deep water waves. Part 1: Unforced irrotational wave groups, J. Phys. Oceanogr., 32, 9, 2541-2558, 2002.

Stokes, G. G.: On the theory of oscillatory waves, Trans. Cambridge Philos. Soc., 8, 441-445, Math. Phys. Pap., 1, 197-229, 1847.

Tanaka, M, Dold, J. W., Lewy, M., and Peregrine D. H.: Instability and breaking of a solitary wave, J. Fluid Mech. 187, 235-248, 1987.

Tanveer, S.: Singularities in water waves and Rayleigh-Taylor instability, Proc. R .Soc., Lond. A435, 137-158, 1991. 
Tanveer, S.: Singularities in the classical Rayleigh-Taylor flow: formation and subsequent motion, Proc. R. Soc. Lond., A441, 501$525,1993$.

Thompson, J. F, Warsi, Z. U. A., and Mastin, C. W.: Boundary-fitted coordinate systems for numerical solution of partial differential equations-a review, J. Comput. Phys., 47, 1-108, 1982.

Trease, H. E., Fritts, M. J., and Crowley, W. P.: Interactions between a free surface and a vortex sheet shed in the wake of a surfacepiercing plate, J. Fluid Mech.257, 691-721, 1990.

Tsai, W. T. and Yue, D. K. P.: Computation of nonlinear free-surface flows, Annu. Rev. Fluid Mech., 28, 249-278, 1996.

Vinje, $\mathrm{T}$ and Brevig, P.: Numerical simulation of breaking waves, Adv. Water Resources, 4, 77-82, 1981.

Watson, K. M. and West, B. J.: A transport-equation description of nonlinear ocean surface wave interactions, J. of Fluid Mech., 70, 815-826, 1975.

West, B. J., Brueckner K. A., and Janda, R. S.: A New Numerical Method for Surface Hydrodynamics, J. Geophys. Res., 92, C11, $11803-11824,1987$.
Whitney, J. C.: The numerical solution of unsteady free-surface flows by conformal mapping, in: Proc. Second Inter. Conf. on Numer. Fluid Dynamics, edited by: Holt, M., Springer-Verlag, 458-462, 1971.

Zakharov, V. E., Dyachenko, A. I., and Vasilyev, O. A.: New method for numerical simulation of a nonstationary potential flow of incompressible fluid with a free surface, European Journ. of Mech. B/Fluids, 21, 283-291, 2002.

Zakharov, V., Dias, F., and Pushkarev A.: One-dimensional wave turbulence, Physics Rep., 398, 1, 1-65, 2004.

Yuen, H. C. and Lake, B. M.: Nonlinear Dynamics of Deep-Water Gravity Waves, Adv. in Appl. Mech., 22, 67-229, 1982.

Yeung, M. A.: Numerical methods in free-surface flows, Ann. Rev. Fluid Mech., 14, 395-442, 1982.

Zhang, J., Hong, K., and Yue, D. K. P.: Effects of wavelength ratio on wave modeling, J. Fluid Mech., 248, 107-127, 1993. 